\title{
Metaheuristic Optimization of LED Locations for Visible Light Positioning Network Planning
}

\author{
Sander Bastiaens (D), Graduate Student Member, IEEE, Sotirios Goudos (D), Senior Member, IEEE, \\ Wout Joseph (D), Senior Member, IEEE, and David Plets (D), Member, IEEE
}

\begin{abstract}
Optical Wireless Communication (OWC) is being explored for application in the next-generation broadcasting networks, where possessing accurately determined user locations becomes increasingly important. Received signal strength (RSS) Visible Light Positioning (VLP)-based localisation systems aim to deliver these centimetre-level location data at a low cost by featuring but a single photodiode (PD). Maximising the VLP accuracy requires optimising the LED transmitter locations, which is missing currently. An evolutionary optimisation algorithm is proposed to determine the optimal LED locations and the associated positioning error values for various configurations. The sensitivity of the planning on the number of VLP-enabled LEDs, the LEDs' characteristics, the room dimensions and the positioning parameters is investigated. Experimental data, i.e. two datasets with $157^{2}$ measurement points each, serve to validate the simulations.
\end{abstract}

Index Terms - Visible Light Positioning, VLP, Positioning, Network Planning, LED locations, Optimization, Evolutionary Algorithm

\section{INTRODUCTION}

By virtue of a vast unregulated and noncongested spectrum, Optical Wireless Communication (OWC) is being researched for application in next-generation (broadcasting) networks [1], [2]. Visible Light Communication (VLC) is a subdomain of OWC, in which illumination Light-Emitting Diodes (LEDs) are leveraged to transmit location-confined data. In seamless conjunction with future radio frequency (RF) technologies, the inherently non-RF-interfering VLC systems are in line to provide a cost-effective last meter solution [3], [4]. In the context of the Internet of Radio Light (IoRL) project [5], [6], VLC is already integrated into a 5G network to ensure connectivity in homes, businesses [7], museums [8] and supermarkets [9].

A user's location has become an important parameter, certainly for several (immersive) broadcasting applications [10], such as wireless augmented and virtual reality [11]. Received signal strength (RSS)-based Visible Light Positioning $(V L P)$-based localisation systems strive to deliver this required,

\footnotetext{
This work is part of the scientific progress made within LEDsTrack. LEDsTrack is a collaboration project between industrial and academic partners, which relies on both imec and Flanders Innovation \& Entrepreneurship for funding and project support.

Sander Bastiaens, Wout Joseph and David Plets are with the research group WAVES, Department of Information Technology (INTEC), Ghent University/imec, Belgium (e-mail: firstname.lastname@ugent.be).

Sotirios Goudos is with the ELEDIA@AUTH, Department of Physics, Aristotle University of Thessaloniki, Greece (e-mail: sgoudo@physics.auth.gr).

Color versions of one or more of the figures in this paper are available online at http://ieeexplore.ieee.org.
}

centimetre-accurate geographic data at a low cost. Parsimonious and higher update rate-requiring RSS-based VLP rollouts generally feature a single photodiode (PD)-based receiver instead of a camera-based one [12].

Unfortunately, current VLP roll-outs of both research and commercial nature do not yet reach the centimetre accuracy requirement set forth by many applications. Li et al. [13] reported a $90^{\text {th }}$ percentile positioning error $p_{90}$ of approximately $25 \mathrm{~cm}$ and $60 \mathrm{~cm}$ respectively with and without the application of sensor fusion in a $2.5 \mathrm{~m}$ by $2.84 \mathrm{~m}$ by $2.5 \mathrm{~m}$ area equipped with 7 LED lamps. Alam et al [14] displayed a $1.9 \mathrm{~cm}$ and a $16.1 \mathrm{~cm}$ median positioning error in respectively a $3.3 \mathrm{~m}$ by $2.1 \mathrm{~m}$ laboratory and a $7.5 \mathrm{~m}$ by $8 \mathrm{~m}$ open foyer environment, when employing spring-relaxation (SR)-based positioning combined with a calibration fit [12]. The practical evaluations of [12] showed a median error $p_{50}$ and a $p_{90}$ of $4.72 \mathrm{~cm} / 5.52 \mathrm{~cm}$ and $9.22 \mathrm{~cm} / 9.39 \mathrm{~cm}$ for two different commercial photodiodes (PDs) in a $4 \mathrm{~m}$ by $4 \mathrm{~m}$ by $3 \mathrm{~m}$ zone. Commercial systems are advocating a $30 \mathrm{~cm}$ accuracy [15].

Scientific literature has identified several high-accuracy hindering factors for VLP [16] such as receiver tilt [17], transmitter tilt [18], non-ideal LED modulation [19], fluctuations in the LEDs' transmit power [20] and the LED's radiation pattern diverging from the tabulated one [21].

The impact of the LED layout on the positioning performance, though important, has not yet been sufficiently studied [22]. In VLP experiments with dedicated VLP rollouts, the LEDs are by default installed in geometrical constellations such as triangles [10], squares or rectangles [12]. Simulation-based works generally consider a standard $2.5 \mathrm{~m}$ spaced square 4 LED arrangement covering a room with dimensions $5 \mathrm{~m} \times 5 \mathrm{~m} \times 3 \mathrm{~m}$ [23]-[25]. Importantly, Plets et al. in [26] already showed the existing position ambiguity found during 3D localisation when Lambertian-like LEDs are placed in a circular constellation. As the cost-effectiveness of VLP rests on it expecting to leverage the existing illumination infrastructure, LED locations can in practice be dictated by the illuminance requirements in the environment.

For VLP to reach its full economic potential, it requires the design of a network planner to both predict a roll-out's installation cost and obtainable tracking accuracy, and to perform automatic constrained design of the VLP installation.

This work provides a first step towards this VLP network planner by investigating the impact of the (Lambertian) VLPenabled LEDs' location on the RSS-based VLP positioning performance. Network planning is a type of optimization 
problem that requires the determination of several geometrical parameters to optimize its performance. Thus, an optimization algorithm is a straightforward approach for solving this problem. Evolutionary algorithms (EAs), as can be found from the literature, have been widely utilized to solve several problems in wireless communications [27].

In this paper, for the first time, an appropriate evolutionary (metaheuristic) optimisation algorithm is employed to determine, i.e. to plan a (sub)optimal VLP roll-out, solely in terms of the positioning error. More specifically, we apply the wellknown Particle Swarm Optimization (PSO) [28], and four emerging algorithms: the Grey Wolf Optimizer (GWO) [29], the JAYA algorithm [30], the Salp Swarm Algorithm (SSA) [31], and the Cat Swarm Optimization (CSO) [32].

This planning algorithm is then wielded to report the (sub)optimal LED locations and their associated positioning error values for various simulation configurations that differ in the number of VLP-enabled LEDs, in the LEDs' characteristics, in the room dimensions, in the positioning parameters, and in the noise magnitude experienced at the $\mathrm{PD}$ receiver. In addition, simulation results are provided to compare the planned and geometrical LED constellations, and to characterise the influence of (small) deviations on the LEDs' placement.

Finally, this work would not be complete without an experimental verification part. Based on the 2 large 2D datasets with 157 measurements per dimension, gathered in our VLP lab [12], the positioning error found when localising with a 'planned' and a square LED layout is reported to demonstrate the importance of well-chosen LED locations. Concretely, the main contributions of this manuscript are:

- The identification of the appropriate LED location planning algorithm, among five of the literature's promising evolutionary algorithms. To the best of the authors' knowledge, this is the first time that these algorithms are applied to this problem.

- A simulation study characterising the positioning accuracy yield that arises from rolling out a VLP system with planned LED locations, amid different environment parameters.

- Experimental verification comparing the planned LED layout with the traditional 'square' LED constellation.

- The various results of this work provide guidelines on the do's and don'ts for VLP planning.

The remainder of this paper is organised as follows. Section II describes the related works, while Section III details the materials and methods utilised in this manuscript. Sections IV and $\mathrm{V}$ discuss the simulation-based planning and appertaining measurement results, respectively. Finally, Section VI] lists the guidelines, conclusions and future work.

\section{RELATED WORKS}

This section lists the exploration works in literature that consider and optimise various LED arrangements and LED characteristics for both VLP and VLC purposes. In VLC, the influence of the LED locations, the LEDs' radiation pattern and the VLC cell (LED beam) radius [33] is usually studied with a focus on signal-to-noise ratio (SNR) and signal-tointerference-plus-noise ratio [34]. In [35], the distance between 2 LEDs is optimised in terms of their area spectral efficiency (ASE) for two receiver field of view (FOV) values. For a $90^{\circ}$ FOV both LEDs were 'pushed' towards the room's boundaries, while for the smaller FOV of $45^{\circ}$ the optimal LED locations shifted significantly inwards towards the room's centre. Singh et al. [36] showed that a VLC system operating with 16 randomly placed LED locations, as obtained with a Matérn hardcore point process, effectuated a more optimal SNR distribution compared to when the 16 LEDs would be placed in a circular or square fashion. Still, an algorithmic 2D optimisation of the LED locations in a practical environment is seldom performed. Only in [37], the authors employ the Fireworks Algorithm to maximise the (average) SNR and the SNR uniformity of 4, 9 and 16 LED deployments in the standard VLP room of dimensions $5 \mathrm{~m}$ x $5 \mathrm{~m} \mathrm{x} 3 \mathrm{~m}$.

The impact of both the LED cell area, in combination with proximity [38], and the LEDs' Lambertian order [39] has also been studied for VLP. The latter demonstrated that VLP systems employing LEDs with an optimal Lambertian order $m$ outscore $m=1$ systems by $10.1 \%, 15.9 \%$ and $15.4 \%$ in terms of their $99^{\text {th }}$ root-mean-square error Cramér-Rao bound (CRB) for a 4, 6 and 9 LED deployment respectively.

The extent of the impact of the LED arrangement on the VLP accuracy has not yet been sufficiently characterised [22]. This is remarkable as improper LED locations can have a detrimental impact on the positioning performance of a VLP system [40]. In [40], Plets et al. demonstrated that the $50^{\text {th }}$ percentile of the positioning error, found during 3D VLP in a $4 \mathrm{~m}$ by $4 \mathrm{~m}$ by $3 \mathrm{~m}$ area, spectacularly increased from $p_{50}=12.7 \mathrm{~cm}$ to $2.26 \mathrm{~m}$ as a consequence of positioning ambiguities [26], when switching from a star LED configuration to a rectangular LED configuration. The LED locations also influence the size of the dead-zones, found during the localisation of unmanned aerial vehicles [41]. Importantly, as to where to place the LEDs to optimise the VLP roll-out's accuracy has also not been addressed in literature. Both open issues will be addressed with the methodology introduced next.

\section{Methodology}

\section{A. Simulator}

\section{1) Localisation}

The 2D VLP positioning accuracy is evaluated in terms of the positioning error, i.e. the Euclidean distance between the actual and estimated receiver position, found when localising an untilted PD-based receiver with an $A_{R}=13 \mathrm{~mm}^{2}$ active area, located on a $10 \mathrm{~cm}$ spaced uniform grid on the $5 \mathrm{~m}$ by $5 \mathrm{~m}$ ground plane, in the presence of $N$ white LEDs that are $3 \mathrm{~m}$ higher up. The LEDs, $\operatorname{LED}_{i}(i=1 . . N)$, have precisely known coordinates $\mathbf{x}_{\mathbf{S}}=\left(x_{S, i}, y_{S, i}, z_{S, i}\right)$ and are intensity modulated to broadcast receiver-side demultiplexable beacon signals with unique frequencies [42]. The received radiant powers $P_{R, i}$, which are the received signal strength (RSS) values appertaining to the LEDs' beacon transmission, serve as inputs to a localisation algorithm to compute a receiver position estimate $\hat{\mathbf{x}}$. 
Multilateration-based VLP converts the set of $P_{R, i}$ first into a set of $\mathrm{LED}_{i}$ - PD distances $d_{i}$ by inverting the VLP channel model of Section III-A2. In a second step, $\hat{\mathbf{x}}$ is obtained via least-squares minimisation of the linearised system relating $\left\{d_{i}\right\}$ to $\mathbf{x}_{\mathbf{S}}$, see e.g. [26], [43] for details. Other VLP positioning algorithms, such as model-based fingerprinting $(M B F)$ [12] will also be considered (in Section [III-B].

Not all LEDs' $P_{R, i}$ (or $d_{i}$ ) are required to compute $\hat{\mathbf{x}}$. In fact, selecting a subset (in terms of descending $P_{R, i} / P_{t, i}$ ) with $K(K<N)$ elements might effectuate a more accurate $\hat{\mathbf{x}}$. $K=3$ is the default value.

\section{2) Propagation}

For strict time-invariant stationary optical channels having a flat frequency characteristic, $P_{R, i}$ can be modelled in terms of the DC channel gain equations of Kahn et al. [44]:

$$
P_{R, i}=\sum_{i=1}^{N} P_{t, i} \cdot h_{c}^{(i)}, \text { with } h_{c}^{(i)}=h_{c, L}^{(i)}+\sum_{A} h_{c, N L}^{(i, d A)}
$$

In (1), for $\mathrm{LED}_{i}, P_{t, i}$ represents the radiant flux/power, whilst the Line-of-Sight (LOS) $h_{c, L}^{(i)}$ and Non-Line-of-Sight (NLOS) $h_{c, N L}^{(i, d A)}$ components (within the receiver field of view $\left|\Psi_{C}\right|$ ) of $h_{c, i}^{(i)}$ amount to:

$$
\begin{gathered}
h_{c, L}^{(i)}=R_{E}\left(\phi_{i}, \gamma_{i}\right) \cdot \frac{A_{R}}{d_{i}^{2}} \cos \left(\psi_{i}\right) \\
h_{c, N L}^{(i, d A)}=\frac{R_{E}\left(\phi_{i}^{\prime}, \gamma_{i}^{\prime}\right) \cos \left(\theta_{i}^{\prime}\right) d A \cos \left(\psi_{i}^{\prime}\right) A_{R} L\left(\theta_{i}, \chi_{i}\right)}{d_{i, 1}^{2} \cdot d_{i, 2}^{2}}
\end{gathered}
$$

$h_{c, N L}^{(i, d A)}$ arises from reflections on, ideally infinitesimally, small surface elements $A$ with area $d A$ present in the scene, e.g. part of walls. In (2) and (3), $d_{i}, d_{i, 1}$ and $d_{i, 2}$ denote the LED-PD, the LED-surface element and surface element-PD distance. $\phi_{i}$, $\phi_{i}^{\prime}, \theta_{i}$ designate respectively the elevation irradiance angle for the LOS and NLOS component, and during reflection. $\gamma_{i} / \gamma_{i}^{\prime}$ symbolise the azimuthal irradiance angles, and $\psi_{i} / \psi_{i}^{\prime} / \theta_{i}^{\prime}$ the incidence angles at the PD and at the surface element. $R_{E}\left(\phi_{i}, \gamma_{i}\right)$ describes the radiation pattern of $\mathrm{LED}_{i}$, which for a Lambertian radiator is characterised by its Lambertian order $m_{i}: R_{E}\left(\phi_{i}, \gamma_{i}\right)=R_{E}\left(\phi_{i}\right)=\left[\frac{m_{i}+1}{2 \pi} \cos ^{m_{i}}\left(\phi_{i}\right)\right]$. Per definition, in multilateration, the LEDs' $R_{E}\left(\phi_{i}, \gamma_{i}\right)$ is assumed to be Lambertian. $L\left(\theta_{i}, \chi_{i}\right)$, the reflected radiation pattern, is governed by Phong's model [45]:

$$
\begin{gathered}
L\left(\theta_{i}, \chi_{i}\right)=\rho \cdot\left(r_{d} \cdot L_{d}\left(\theta_{i}\right)+\left(1-r_{d}\right) \cdot L_{s}\left(\chi_{i}\right)\right) \\
L_{d}\left(\theta_{i}\right)=\left(\frac{m_{d}+1}{2 \pi}\right) \cos ^{m_{d}}\left(\theta_{i}\right), L_{s}\left(\chi_{i}\right)=\left(\frac{m_{s}+1}{2 \pi}\right) \cos ^{m_{s}}\left(\chi_{i}\right)
\end{gathered}
$$

$L_{d}\left(\theta_{i}\right)$ and $L_{s}\left(\chi_{i}\right)$ describe the NLOS' diffuse and specular part. $r_{d}, m_{d}, m_{s}, \rho, \chi_{i}$ equal the fraction of diffusivity, the Lambertian orders of the diffuse and specular component, the reflection coefficient, and the enclosed angle between the specular ray and the PD- $A$ vector, respectively.

\section{3) System Parameters in Simulation}

To match the simulations with the subsequent experimental validation, most of the PD and LED parameters are taken to be those of the devices utilised in the measurement setup (Section III-C). The LEDs' radiant powers satisfy: $P_{t, i}=$
$P_{t}=10 \mathrm{~W}$. The noise sources found in the photocurrent generated at the PD (and that hence bedevil $P_{R, i}$ ) are modelled in terms of an input-referred current noise, which is assumed to be both additive and Gaussian with a zero mean and a $\sigma_{I}^{2}$ variance. The default $\sigma_{I}$ value is taken from the photocurrent measurements performed by Plets et al. [40]. However, a reweighting with the LED-PD wavelength mismatch factor $M$ [12] is performed as in this work a different LED type (i.e. emission spectrum) is used. Multiplying the previous with the receiver responsivity results in $\sigma$, dictating the noise component on $P_{R, i}$. Furthermore, per location estimate, 10 $P_{R, i}$ measurements are averaged to reduce the noise impact.

\section{B. LED Location Planning/Optimisation}

As stated in the introduction, an evolutionary optimisation algorithm will be wielded for determining the 'optimal' LED arrangement for environments differing in number of VLPenabled LEDs, in LED characteristics, in room dimensions, and in the noise magnitude experienced at the PD receiver. Five algorithms are considered: Particle Swarm Optimization (PSO) [28], the Grey Wolf Optimizer (GWO) [29], the JAYA algorithm [30], the Salp Swarm Algorithm (SSA) [31], and the Cat Swarm Optimization (CSO) [32]. The basic details of each of the algorithms are briefly described below.

\section{1) $\mathrm{PSO}$}

Particle Swarm Optimization (PSO) with inertia weight $[28]$ is the original and most popular $P S O$ algorithm. The fundamental concept of $P S O$ is the movement of a swarm of particles in the search space. $P S O$ uses the information taken from two optimum values to modify each particle's position in every iteration. The first optimum is the best solution (objective function value) that has been achieved so far by each particle in the swarm. This optimum is denoted as pbest and is called personal best. The second optimum is the global best value obtained by any particle in the swarm. This optimum value is denoted as gbest. Thus, for every iteration, PSO finds these pbest and gbest values. Additionally, a velocity update rule that models swarm movement is used. It plays an important role in obtaining gbest, and for the $k^{\text {th }}$ particle in the $m^{\text {th }}$ dimension it is given by:

$$
\begin{aligned}
u_{G+1, m k}=w \cdot u_{G, m k} & +c_{1} \cdot \operatorname{rand}_{1, m k}\left(\text { pbest }_{G+1, m k}-x_{G, m k}\right) \\
& +c_{2} \cdot \operatorname{rand}_{2, m k}\left(\text { gbest }_{G+1, m k}-x_{G, m k}\right)
\end{aligned}
$$

where $u_{G+1, m k}$ denotes the $k$-th particle velocity in the $m$-th dimension, $G+1, G$ represent the subsequent and the current iteration respectively, $x_{G, m k}$ is the $k$-th particle position in the $m$-th dimension, in the current iteration, $\operatorname{rand}_{1, m k}, \operatorname{rand}_{2, m k}$ are uniformly distributed random numbers in $[0,1], w$ is the inertia weight, $c_{1}$, and $c_{2}$ are called the learning factors. The new particle position in the $(G+1)^{\text {th }}$ iteration of the $k^{\text {th }}$ particle in the $m^{\text {th }}$ dimension is $x_{G+1, m k}$ and is derived by:

$$
x_{G+1, m k}=x_{G, m k}+u_{G+1, m k}
$$

The PSO algorithm requires setting three control parameters: the inertia weight $w$ and the positive constants $c_{1}, c_{2}$. The inertia weight $w$, commonly $w \in[0,1]$, represents the particle's motion without any external influence. The higher the 
value of $w$, the more the particle stays unaffected from pbest and gbest. Hence, $w$ controls the exploration and exploitation of the search space, where large inertia weight values favour exploration and small $w$ values favour exploitation.

2) $G W O$

The $G W O$ algorithm is based on the mathematical modelling of the basic mechanisms (hierarchy and hunting) of grey wolves in nature [29]. Its main characteristic is the preservation of information about the search space over the iteration process. In $G W O$, it is not required to set any control parameters. The $G W O$ algorithm divides the population into four categories. The first three best solutions/grey wolves are deemed the alpha $(\alpha)$, beta $(\beta)$, and delta $(\delta)$ categories. All the unclassified solutions are grouped into the omega $(\omega)$ category. Thus, the group hunting (i.e. the optimisation process), a social behaviour in a pack of wolves, is oriented by the aforementioned categories $(\alpha, \beta, \delta)$ of the population. The mathematical description of the prey encirclement in the hunting process is given by the following expressions:

$$
\begin{gathered}
\vec{V}=\left|\vec{C}^{2} \cdot \vec{P}_{G}-\vec{W}_{G}\right| \\
\vec{W}_{G+1}=\vec{P}_{G}-\vec{C}^{1} \cdot \vec{V}
\end{gathered}
$$

where $\vec{C}^{1}$ and $\vec{C}^{2}$ are coefficient vectors, $\vec{P}$ is the position vector of the prey, $\vec{W}$ corresponds to the position vector of the grey wolf, and $G$ indicates the current generation. The vectors $\vec{C}^{1}$ and $\vec{C}^{2}$ are given by $(10)$ and $(11)$ :

$$
\begin{gathered}
\vec{C}^{1}=2 \vec{u} \cdot \vec{v}_{1}-\vec{u} \\
\vec{C}^{2}=2 \cdot \vec{v}_{2}
\end{gathered}
$$

where $\vec{v}_{1}, \vec{v}_{2}$ are random vectors in $[0,1]$, and the components of $\vec{u}$ are linearly decreasing from 2 to 0 with an increasing iteration number. The hunting process of a grey wolves' pack can then be described by the following set of equations.

$$
\begin{aligned}
& \vec{V}_{\alpha}=\left|\vec{C}_{1}^{2} \cdot \vec{W}_{\alpha}-\vec{W}\right| \\
& \vec{V}_{\beta}=\left|\vec{C}_{2}^{2} \cdot \vec{W}_{\beta}-\vec{W}\right| \\
& \vec{V}_{\delta}=\left|\vec{C}_{3}^{2} \cdot \vec{W}_{\delta}-\vec{W}\right| \\
& \vec{W}_{1}=\vec{W}_{\alpha}-\vec{C}_{1}^{1} \cdot\left(\vec{V}_{\alpha}\right) \\
& \vec{W}_{2}=\vec{W}_{\beta}-\vec{C}_{2}^{1} \cdot\left(\vec{V}_{\beta}\right) \\
& \vec{W}_{3}=\vec{W}_{\delta}-\vec{C}_{3}^{1} \cdot\left(\vec{V}_{\delta}\right) \\
& \vec{W}_{G+1}=\frac{\vec{W}_{1}+\vec{W}_{2}+\vec{W}_{3}}{3}
\end{aligned}
$$

3) Jaya

Jaya is a very simple algorithm having a low complexity and was first introduced in [30]. Jaya uses as main concept the mathematical principle that at each iteration each solution vector should be updated in order to move towards the best, and away from the worst solution obtained. The algorithm name's origin is found in the Sanskrit word "Jaya", which means victory.

Jaya does not require the setting of control parameters. It updates the position of each member of the population using the best $\mathbf{y}_{\mathbf{b}}$ and the worst $\mathbf{y}_{\mathbf{w}}$ solution vector at each iteration. Hence, the $k^{\text {th }}$ member of the population $\mathbf{y}_{\mathbf{k}}$ updates its position with the following rule:

$$
\mathbf{y}_{\mathbf{i}}^{\text {new }}=\mathbf{y}_{\mathbf{i}}^{\text {old }}+r n d_{1}\left(\mathbf{y}_{\mathbf{b}}-\mathbf{y}_{\mathbf{i}}^{\text {old }}\right)-r n d_{2}\left(\mathbf{y}_{\mathbf{w}}-\mathbf{y}_{\mathbf{i}}^{\text {old }}\right)
$$

where $r n d_{1}$ and $r n d_{2}$ are uniformly distributed random numbers within the range $[0,1]$. Jaya uses a greedy selection operator.

4) $S S A$

SSA mathematically models the swarming behaviour of salps that navigate and forage in oceans [31]. In $S S A$, each salp is a $D$-dimensional solution vector. The salp (i.e. the solution vector) that obtains the best objective function value per iteration represents the food position.

In $S S A$, the salps' behaviour is modelled by partitioning the population into two groups. The salps in-front of the salp chain are called the leaders. The rest of the salps are called the followers. The leaders are the salps with the best objective function values, while the salps with the inferior function values are considered to be the followers.

$S S A$ divides the population into two groups of equal size $\frac{N P}{2}$, where $N P$ denotes the population size. The leaders' positions satisfy the update rule below:

$$
x_{G+1, m, k}=\left\{\begin{array}{l}
F_{k}+C_{1} \times\left[y_{k}\right], r_{3 k}<0.5 \\
F_{k}-C_{1} \times\left[y_{k}\right], r_{3 k} \geq 0.5 \\
y_{k}=r_{2 k}\left(x_{k, U}-x_{k, L}\right)+x_{k, L}
\end{array}\right.
$$

where $x_{G+1, m, k}$ denotes the position of the $m^{\text {th }}$ leader salp with $m<N P / 2$ in the $k^{\text {th }}$ dimension for generation $G+1, F_{k}$ represents the food source position (or the best vector in the population) in the $k^{\text {th }}$ dimension, $x_{k, U}, x_{k, L}$ are the upper and lower bounds in the $k^{\text {th }}$ dimension respectively. The parameter $C_{1}$ is an important one for $S S A$ and is defined by:

$$
C_{1}=2 \times e^{-\left(\frac{4 G}{G_{\max }}\right)^{2}}
$$

where $G$ is current iteration and $G_{\max }$ the maximum number of iterations. According to [31], the importance of the $C_{1}$ parameter lies in the fact that it balances exploration and exploitation. Additionally, $r_{2 k}, r_{3 k}$ are uniform distributed random numbers within the interval $[0,1]$. These random numbers help the algorithm to decide both whether the next position in $k^{\text {th }}$ dimension will be towards positive infinity or negative infinity, and the step size. SSA defines a different position update rule for the other $\frac{N P}{2}$ vectors (i.e. the followers):

$$
x_{G+1, m, k}=\left(x_{G, m, k}+x_{G, m-1, k}\right) / 2
$$

\section{5) $\mathrm{CSO}$}

CSO models the behaviour of the cats. The cats' positions are potential solutions to the optimisation problem. $C S O$ works with two modes: the seeking and the tracing mode. In these modes, the cats' positions are updated in different ways.

At first, after initialization, the cats are randomly classified to either tracing or seeking mode, according to a mixing ration $(M R)$ parameter. Subsequently, the fitness value of all cats is calculated, with the best one kept in memory. Afterwards, the cats move as dictated by their mode. In the next iteration, the cats are again randomly classified in tracing and seeking. This process continues until the termination criterion is met. 
CSO defines the following control parameters: the seeking memory pool $(S M P)$, the seeking range of the selected dimension $(S R D)$, the counts of dimension to change $(C D C)$, and the self-position considering ( $S P C)$. SMP defines the size of the seeking memory for each cat. Moreover, $S R D$ declares the mutative ratio for the selected dimension(s). $C D C$ shows the number of position dimensions that will be varied. The role of these parameters is important in the seeking mode. SPC is a Boolean variable that denotes if the point where the cat is already located, will be one of the candidates to move to. The authors of $C S O$ discuss, in [32], the advantages of CSO over other well-known bio-inspired algorithms (such as $P S O$ ).

\section{6) Control parameter setting}

In all algorithms, the population size is set equal to 20 , while the maximum number of objective function evaluations is set to 400. Initialisation is random. Three algorithms, namely GWO, SSA and Jaya, are parameter-free, i.e. they do not require the setting of any additional parameters. The other algorithms' control parameters have been set based on the parameter settings found in the literature that presumably perform well for an arbitrary problem. For PSO, both the $c_{1}, c_{2}$ parameters amount to 2 , while the inertia weight is linearly decreased with each iteration from $w_{\max }=0.9$ to $w_{\min }=0.2$. For $C S O$, the parameters satisfy: $S M P=5$, $S R D=0.2, C D C=0.8, M R=0.02$ (meaning that $2 \%$ of the cats are in seeking mode) and $S P C=$ false.

7) Objective Function Minimising the Positioning Metric

For the purpose of VLP network planning, the (single-) objective function the 5 previous algorithms optimise consists of minimising the positioning error over the positioning grid (see Section III-A by varying the LEDs' coordinates. Specifically, these algorithms are applied to minimise the $p_{10-90}$ metric, which is defined as the area confined between $10 \%$ and $90 \%$ underneath the quantile function:

$$
p_{10-90}=\int_{0.1}^{0.9} C D F(y)^{-1} d y
$$

with $C D F(y)^{-1}$, the inverse of the cumulative distribution function (CDF) of the positioning error on the grid. The position error itself is defined as the Euclidean distance between the ground truth position and its location estimate. $p_{10-90}$ is chosen as to cope with positioning outliers, and is 20 times averaged (20 independent positioning trials) per objective function evaluation.

In conclusion, each optimisation algorithm's outputted LED locations minimise the $p_{10-90}$, whilst satisfying the enforced positioning area's boundary conditions.

\section{Measurement Setup}

To verify the eventual network planner, consisting of the best performing optimisation algorithm of Section III-B experimental localisation data will be gathered in our $4 \mathrm{~m}$ by $4 \mathrm{~m}$ VLP lab [12]. There, the LED plane is occupied by $4 B X R E$ 35E2000-C-73 Chip on board (COB) LEDs, modulated by

https://www.bridgelux.com/sites/default/files/resource_media/Bridgelux\%20DS101\% 20Gen\%207\%20V13\%20Array\%20Data\%20Sheet\%2020190930\%20Rev\%20N.pdf

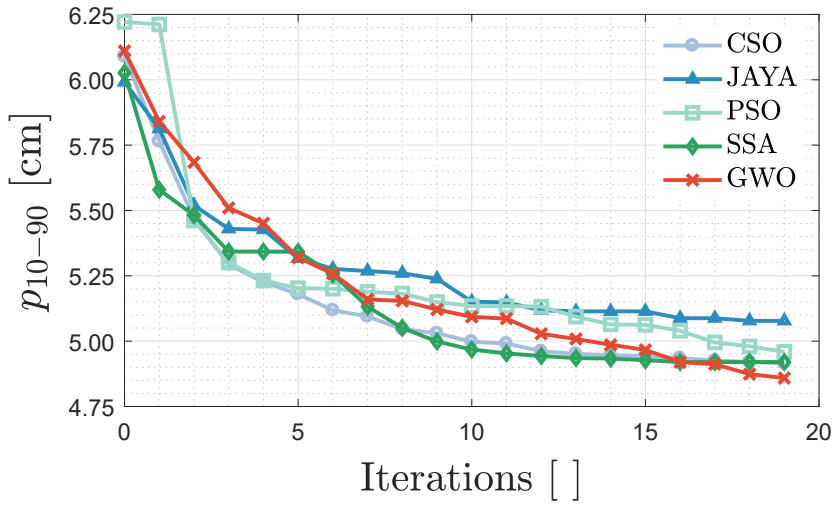

Fig. 1. $p_{10-90}$ versus the algorithm's iteration number for each of the 5 planning algorithms of Section III-B

LTM8005 Demo Board\$2 effectuating the square wave-based frequency-division multiple access (FDMA) scheme [42] with frequencies $f_{c, i}=2^{i-1} f_{0}(i=1 . .4)$ dictated over Wi-Fi via the Adafruit Feather M0 WIFI w/ATWINC1500 ${ }^{3}, f_{0}$ is taken to be $1 \mathrm{kHz}$ as not to induce flicker. The receiver plane, separated $3 \mathrm{~m}$ from the LEDS, is every $2.5 \mathrm{~cm}$ traversed by a Thorlabs PDA36A2 $2^{4}$ PD (with a transimpedance gain set to $1.51 \cdot 10^{5} \mathrm{~V} / \mathrm{A}$ ) placed on top of $2 \mathrm{D}$ Velmex' BiSlides. The resulting photovoltage is measured for a number of samples $N=2560 \mathrm{~S}$ at a rate $f_{S}=256 \mathrm{kHz}$ and then demodulated into RSS values $P_{R, i}$ as outlined in [40].

\section{Planning and Simulation Results}

\section{A. LED Location Optimisation in standard VLP Conditions}

Before obtaining the (sub)optimal LED locations for differing VLP roll-outs, this first result section initially focusses on the standard VLP configuration, which consists of 4 LEDs with order $m=1$ covering a $5 \mathrm{~m}$ by $5 \mathrm{~m}$ area located $3 \mathrm{~m}$ below. It will identify the most suitable single-objective $L E D$ location planning algorithm among those of Section III-B Once identified, this algorithm will be utilised to study the LED locations' impact on the RSS-based VLP positioning error, found in various environments. In this section, the variance and optimality of the planned LED locations will be investigated as well. The planned $p_{10-90}$ is compared to the $p_{10-90}$ of common, geometrical LED constellations.

\section{1) Identifying the Appropriate Planning Algorithm}

Fig. 1 depicts the $p_{10-90}$ convergence graph of each the 5 planning algorithms, when up to 20 iterations are run for the standard VLP roll-out with $K=3$ used for positioning. Each algorithm's relation between the $p_{10-90}$ and the iteration number is averaged over 10 different (planning) runs. Table I furthermore lists the best and median $p_{10-90}$, and the standard deviation (SD) on $p_{10-90}$ over the 10 runs.

Fig. 1 and Table 1 provide the rationale for selecting the GWO algorithm for planning the subsequent VLP roll-outs. After all, the best and the lowest median $p_{10-90}$ appertains to $G W O$. While the second to fourth ranked algorithms, namely

\footnotetext{
2 https://www.analog.com/en/products/ltm8005.html\#product-overview

https://www.adafruit.com/product/3010

https://www.thorlabs.com/thorproduct.cfm?partnumber=PDA36A2
} 
TABLE I

THE $p_{10-90}$ SCORES IN CENTIMETRE OF THE 5 PLANNING ALGORITHMS AFTER 20 ITERATIONS

\begin{tabular}{c|c|c|c|c|c|c} 
& Best & Median & SD & $\overline{\text { Best }}$ & $\overline{\text { Median }}$ & $\overline{\text { SD }}$ \\
\hline CSO & 4.87 & 4.91 & 0.04 & 4.88 & 4.93 & 0.05 \\
GWO & 4.81 & 4.84 & 0.06 & 4.86 & 4.92 & 0.05 \\
JAYA & 4.94 & 5.05 & 0.12 & 5.02 & 5.05 & 0.13 \\
PSO & 4.86 & 4.96 & 0.09 & 4.88 & 4.99 & 0.10 \\
SSA & 4.84 & 4.92 & 0.05 & 4.93 & 5.02 & 0.06 \\
\hline
\end{tabular}

SSA, CSO, PSO, all perform comparably, JAYA scores distinctively inferior. Furthermore, whereas the $p_{10-90}$ accuracy benefit of additional SSA, CSO and JAYA iterations saturates, $G W O$ (and $P S O$ ) are not converging at iteration number 19. With the exception of a longer-taking $C S O$, the execution times of the planning algorithms are roughly equal.

Table [I also lists the average of the algorithm's planned outputs' best $(\overline{\mathrm{Best}})$ and the median $(\overline{\text { Median }}) p_{10-90}$ scores across 1000 (instead of 20) independent positioning runs (to reduce the noise impact). Besides confirming the previous conclusions, this shows that the $p_{10-90}-$ gap between $G W O$ 's and CSO's median planning solution has narrowed, albeit it with $C S O$ 's execution time being distinctively longer. Hence, as $G W O$ supplies both the most optimal overall and median solution, it is the algorithm selected to plan with.

2) Optimality and Shape of the GWO LED constellation

This section investigates $G W O$ 's actual planning results i.e. the LED transmitter locations. Hereto, Fig. 2 visualises the two best LED constellation outputs of $G W O$, respectively denoted by $G W O-1$ and $G W O-2$, in black and in the dark blue shade. Both solutions are quadrilaterals not possessing, but neighing to having, additional symmetrical properties e.g. $G W O-1$ is an isosceles trapezoid to within $3.66 \%$.

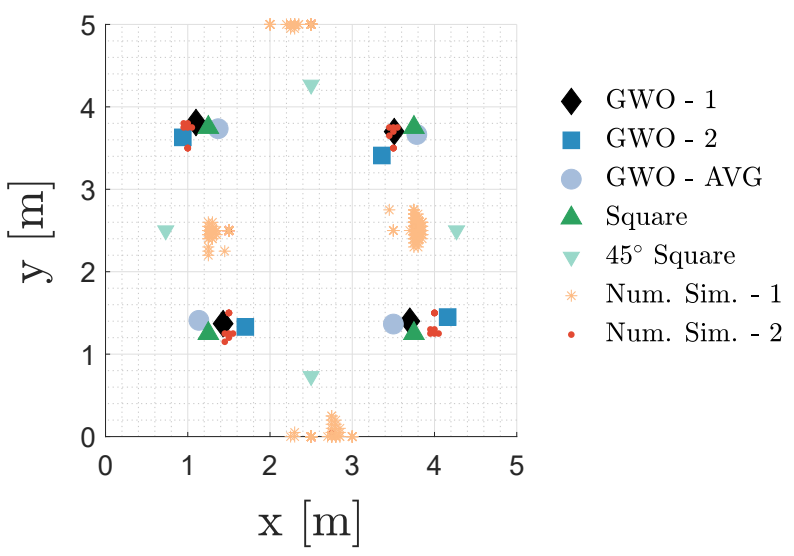

Fig. 2. Spatial LED location distribution for various LED constellations.

The hypothesis that $G W O$ finds the $p_{10-90}$ optimal LED locations is quickly refuted. The suboptimality of $G W O-1$ is demonstrated by it being outscored by the best-performing average, in terms of $p_{10-90}$, of $G W O-1$ and the set of roomsymmetrically rotated/mirrored $G W O-2$ solutions. The shape of this average, dubbed $G W O-A V G$, resembles a parallelogram by virtue of only exhibiting a $24.5 \mathrm{~cm}$ difference between the sides of the parallelogram law equation. The mean $p_{10-90}$
TABLE II

$p_{10-90} / p_{50} / p_{75} / p_{90}$ AND THE ENCLOSED AREA $A$ FOR VARIOUS LED ARRANGEMENTS, AVERAGED ACROSS 1000 POSITIONING SIMULATIONS FOR STATISTICAL SIGNIFICANCE.

\begin{tabular}{|c|c|c|c|c|c|}
\hline Description & $p_{10-90}[\mathrm{~cm}]$ & $\boldsymbol{p}_{\mathbf{5 0}}[\mathrm{cm}]$ & $\boldsymbol{p}_{75}[\mathrm{~cm}]$ & $\boldsymbol{p}_{\mathbf{9 0}}[\mathrm{cm}]$ & $\boldsymbol{A}\left[m^{2}\right]$ \\
\hline GWO - 1 & 4.859 & 5.27 & 9.25 & 14.66 & 5.54 \\
\hline GWO - 2 & 4.876 & 5.24 & 9.33 & 15.06 & 5.56 \\
\hline GWO - AVG & 4.852 & 5.27 & 9.23 & 14.61 & 5.54 \\
\hline Square & 4.874 & 5.30 & 9.27 & 14.61 & 6.25 \\
\hline $45^{\circ}$ Square & 5.063 & 5.32 & 9.64 & 16.98 & 6.25 \\
\hline Num. Sim. - 1 & 4.847 & 5.11 & 9.29 & 15.80 & 5.63 \\
\hline Num. Sim. - 2 & 4.868 & 5.13 & 9.34 & 15.91 & 5.75 \\
\hline
\end{tabular}

across 1000 simulations of $G W O-1, G W O-2$ and $G W O-A V G$ are collected in Table III. Table II lists the $50^{\text {th }} p_{50}, 75^{\text {th }} p_{75}$ and $90^{\text {th }} p_{90}$ percentile positioning error and the area enclosed between the 4 LEDs as well.

From Table II, it can be seen that $G W O-A V G$ reduces $G W O$ - 1's $p_{10-90}$ from $4.86 \mathrm{~cm}$ to $4.85 \mathrm{~cm}$. Though a small (but statistically significant) $p_{10-90}$ difference, it can be concluded that the Grey Wolf-based planning algorithm typically will not have converged towards the optimal LED locations, certainly not after the finite amount of 20 iterations. In accordance to Table I] Table II displays a noteworthy $p_{10-90}$ difference between the two different $G W O$-solutions, thereby attesting to the (substantial) variance in optimality of the $G W O$-planned solutions. In the following, references to the $G W O-1$ solution will be shortened to $G W O$.

Table II also lists the $p_{10-90}, p_{50}, p_{75}$ and $p_{90}$ scores associated with the standard $2.5 \mathrm{~m}$-side square LED arrangement Square and its $45^{\circ}$ rotated variant $45^{\circ}$ Square. Both LED constellations are visualised in green in Fig. 2 as well.

With the exception of an (approximately) equal $p_{90}$, the preeminent $G W O$-planned roll-out (slightly) outperforms Square (and certainly $45^{\circ}$ Square). However, it can be remarked that the gap between both progressively narrows with an increasing positioning metric. Fig. 3 (a) and (d) substantiate the former conclusion by depicting the error contour plot of Square and $G W O$, respectively. The figures show the spatial regions of enhanced or degraded performance. In $G W O$, the skewing of the LEDs with respect to Square, slightly enlarges the area of good, i.e. with a low error, performance at the cost of larger errors in the the room's corners. Importantly though, the square LED arrangement beats most of the $10 \mathrm{GWO}$ solutions (after 20 iterations).

3) Positioning Accuracy of common LED Constellations

To further explore the LED arrangement space, Fig. 3 also portrays the positioning error contours for LEDs organised in two other prevalent, geometrical configurations: namely the star (Fig. 3 (b)) and the triangle (Fig. 3 (c)). In both, the adherent LEDs are separated by $2.5 \mathrm{~m}$. The centre of gravity of the triangle coincides with the room's centre. A comparison of the subfigures of Fig. 3 demonstrates the importance of the LED arrangement on the positioning error as nor the $2.5 \mathrm{~m}$-sided triangle, nor the $2.5 \mathrm{~m}$-spaced star configuration effectuate a $p_{10-90}$ improvement. The $p_{10-90}$ scores of the 3 common (with the square), geometrical LED constellations are graphically collected in Fig. 4. 


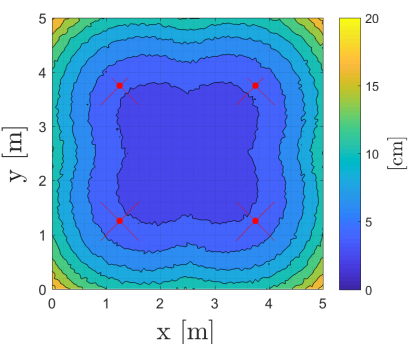

(a)

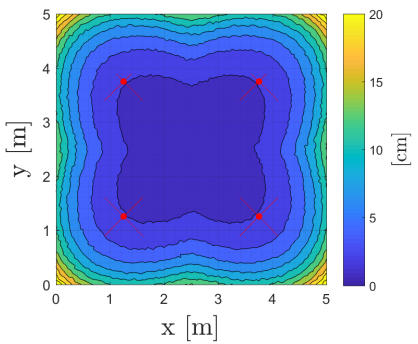

(e)

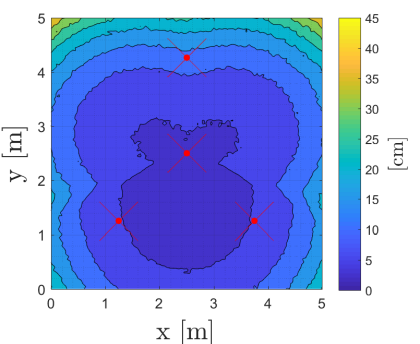

(b)

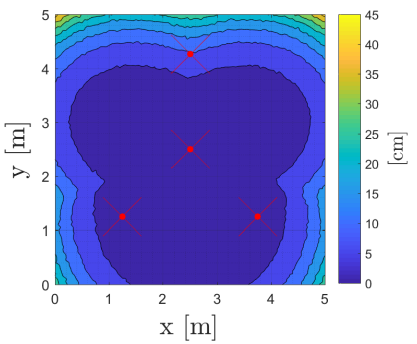

(f)

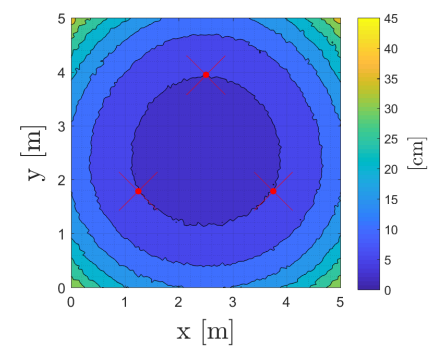

(c)

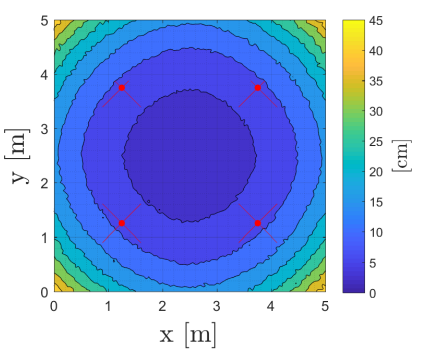

(g)

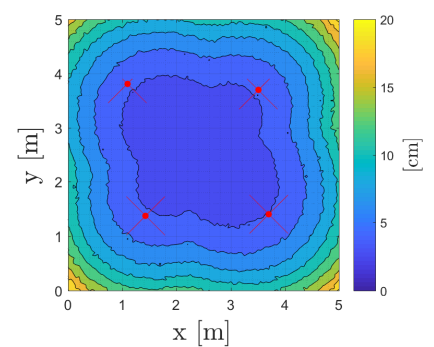

(d)

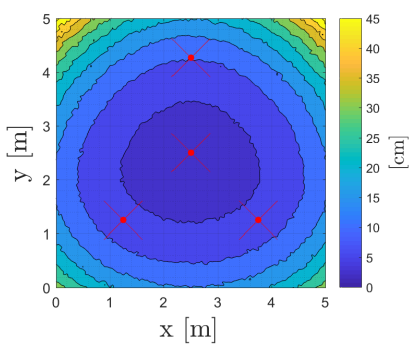

(h)

Fig. 3. Contour plots of the positioning error for the (a) square, (b) star, (c) triangle and (d) planned LED arrangement with $m=1$ and $K=3$, for the (e) square and (f) star LED arrangement with $m=5$ and $K=3$, and for the (g) square and (h) star LED constellation with $m=1$ and $K=4$. Please note the different colour scale of the contour plots. $K$ and $m$ denote the number of LEDs selected for positioning and the LEDs' Lambertian order, respectively.

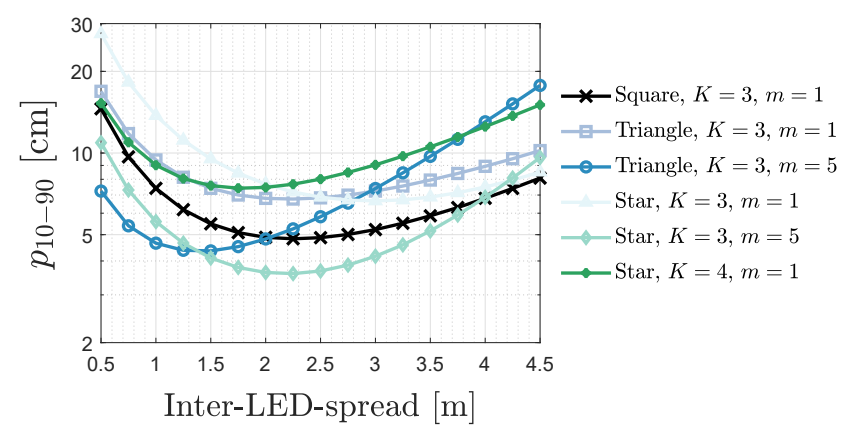

(a)

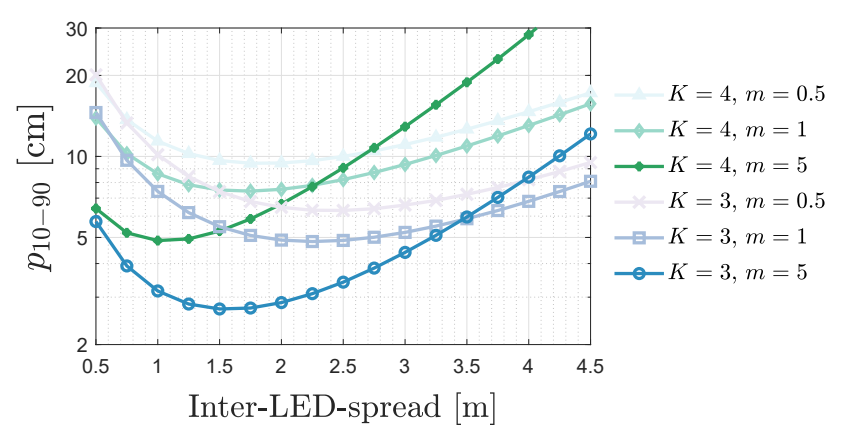

(b)

Fig. 4. $p_{10-90}$ versus the inter-LED-spread, (a) comparing the square, star and triangle arrangement, and (b) visualising the impact of the $m$ and $K$ parameter for LEDs placed in the corners of a square. It should be noted that LEDs, in the star and triangle formation at large inter-LED-spread values, are having coordinates outside of the positioning grid/room and merely serve as reference.

Fig. 4 (a) visualises the $p_{10-90}$ (for $K=3$ and $m=1$ ) for the square (in black), triangular (light blue) and star-shaped (light green) LED arrangement as a function of their interLED-spread, which is the separation between the adherent LEDs. For 2D RSS-based VLP, Fig. 4 proves that, out of the 3 common LED arrangements, it is beneficiary to position the LEDs in a square fashion. Important herewith is to state that only 2D localisation is considered here. In fact, Plets et al. already demonstrated using experimental data that this is not the case for 3D VLP [26] due to positioning ambiguities.

Fig. 4 also shows the optimal inter-LED-spread for the square, star and triangle configuration to amount to $2.25 \mathrm{~m}$, $3 \mathrm{~m}$ and $2.25 \mathrm{~m}$, respectively. Most notably, a square LED constellation with sides $2.25 \mathrm{~m}$ evinces the lowest $p_{10-90}$ score of $4.83 \mathrm{~cm}$. The corresponding $p_{50}, p_{75}$ and $p_{90}$ equal respectively $5.25 \mathrm{~cm}, 9.18 \mathrm{~cm}$ and $14.50 \mathrm{~cm}$, all of which are besting the associated $G W O$ metrics. Rotation of the $2.25 \mathrm{~m}$ square, analogous to $45^{\circ}$ Square hereinabove, will reduce the $p_{10-90}$ performance. In fact, starting from LEDs in a square, the $p_{10-90}$ influence of rotating with angle $\theta$ can be described with $A+B \cdot \sin (4 \theta-\pi / 2)$ (with $\mathrm{A}$ and $\mathrm{B} \sigma$-dependent constants).

\section{4) GWO versus a Discrete Search}

A fine-grained, exhaustive search towards the $p_{10-90}$ optimal LED locations is a hopeless task. However, a discrete numerical search provides insight in GWO's performance. This search consists of 3 steps: (1) Computing the $p_{10-90}$ for all combinations of 4 LEDs on a coarse $25 \mathrm{~cm}$ grid, using only a select number of simulations. (2) Extending the search space with a $5 \mathrm{~cm}$ mesh around the 200 best scoring solutions of step (1) and executing 200 simulations to determine the $p_{10-90}$. (3) Selecting the best 200 LED arrangements from the second step, and calculating their final $p_{10-90}$ over 1000 runs.

Fig. 2 depicts exemplar locations of the two premier classes of solutions obtained with the discrete search, Num. Sim. -1 and Num. Sim. -2 , in orange and red. As can be seen, 
the discrete search missed the $2.25 \mathrm{~m}$-square as well. Still, Num. Sim. -1 improves GWO's best $p_{10-90}$ (see Table III). However, this $p_{10-90}$ reduction does not warrant the order of magnitude larger searching time. Furthermore, Num. Sim. -1 requires the LEDs to be placed against the wall, which proves difficult to realise in practice. The second class of solutions, Num. Sim. -2 , corresponds to the type of arrangement $G W O$ outputs. As $G W O$ is significantly faster, it is the most-suited for the purpose of network planning.

Note that Num. Sim. -1 outscoring the $2.25 \mathrm{~m}$ square arrangement in terms of its $p_{50}$, indicates the dependence of the positioning metric on the (optimality) of the LED locations.

5) Summary of GWO for the standard roll-out

The extensive analysis of this Section IV-A demonstrated that the best and median (from 10) $G W O$-planned LED configuration, respectively, vastly outscores and matches, the traditional $2.5 \mathrm{~m}$ square LED layout in terms of the $p_{10-90}$. However, in the standard $5 \mathrm{~m} \times 5 \mathrm{~m} \times 5 \mathrm{~m}$ room, appropriately sizing the LED square distances leads to a more optimal solution than $G W O$. GWO does approach the former's $p_{10-90}$ to well within $1 \mathrm{~mm}$.

For multilateration-based VLP in traditional LOS environments, GWO's (potentially) obtainable $p_{10-90}$ gain will probably not be worth the additional installation effort or cost, when compared to the square LED constellation. However, the GWO algorithm will in particular have its merit in planning the (sub)optimal LED (lattice) locations for arbitrary environments that are potentially influenced by significant NLOS components, where the optimal multilateration solution will not be square [46]. Finally, increasing the spatial resolution of the positioning grid, will lead to decreasing positioning metrics (a doubling reduces the metrics by approximately 1-3\%).

\section{B. Influence of various Positioning Parameters}

This section extends the previous analysis, of the standard 4 LED $m=1$ VLP roll-out with $K=3$, to cover the influence of $m$, the number of LEDs $N$, the positioning algorithm, $K$ and $\sigma$. In the following, at each time, $1 G W O$ run is performed per data point.

\section{1) Influence of noise on the Planning}

The quality (pre-amplifier noise) of the PD-based receiver (and to a lesser extent of the LED transmitter), and the illuminance (distribution) level of ambient light (originating from sunlight or spurious VLP signal components), dictate the standard deviation $\sigma$ of the $P_{R, i}$ noise component experienced during VLP operation [47]. Hence, as $\sigma$ may vary, the influence of $\sigma$ on the $G W O$-output locations needs to be investigated. Fig. 5 shows an approximately linear relation between the $\sigma$ operating level and the $p_{10-90}$ for various $K$ and $m$ values. The $p_{10-90}-\sigma$ ratio is related to the (horizontal) dilution of precision (treated with measurements in [48]).

Whereas in the former $G W O$ is rerun for each noise value considered (in darkest blue), for $m=1$ and $K=3$, the quasi-overlapping $p_{10-90}$ curve appertaining to the best $G W O$ solution of Section IV-A (i.e. planned for the standard $\sigma \approx 12 \mathrm{nW}$ ) attests that this replanning could be redundant.

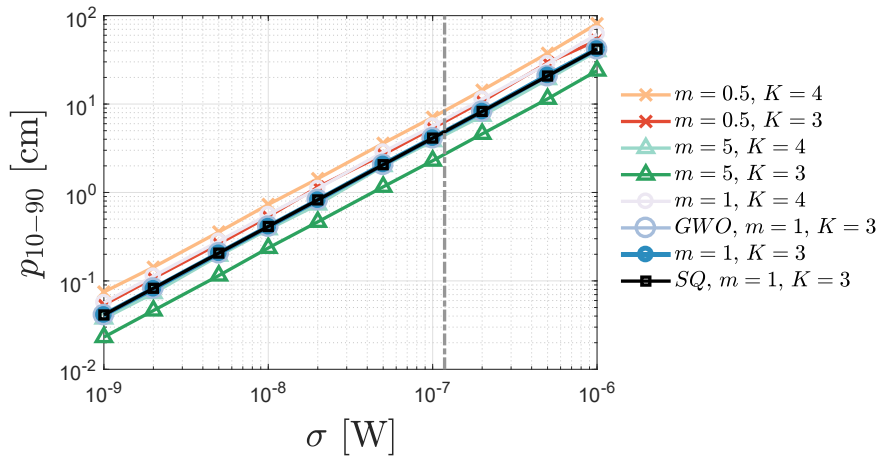

Fig. 5. Influence of the magnitude of the noise's standard deviation $\sigma$ on the $p_{10-90}$ for $G W O$-planned locations with $K \in[3,4], m \in[0.5,1,5]$, and for the best $G W O$ solution from Section IV-A (denoted by $G W O$ ) and the $2.5 \mathrm{~m}$ square (denoted by $S Q$ ) with $K=3$ and $m=1$. The standard $\sigma$ value of $\sigma \approx 12 \mathrm{nW}[40]$ is indicated by the vertical grey line.

The latter $\sigma-p_{10-90}$ curve is shown in the lightest blue in Fig. 5. Errors, introduced by selecting a suboptimal set of $K$ $\left\{P_{R, i}\right\}$ lie within the range of GWO's variance. The GWObased solutions generally (marginally) outscore the reference square LED constellation (denoted by $S Q$ and depicted in black) in the practical $\sigma$ operating range.

2) Influence of $m$

Fig. 5 also depicts the $p_{10-90}$ reduction found in the $5 \mathrm{~m}$ x $5 \mathrm{~m} \mathrm{x} 3 \mathrm{~m}$ room for both $K=3$ and $K=4$, when increasing the Lambertian order from $m=0.5(57.1 \%)$ or $m=1(44.2 \%)$ to $m=5$. An increased $m$ induces an elevated gradient in the $P_{R, i}-d_{i}$ relation. This results in an accuracy improvement closer to the LEDs, accompanied by a deterioration in the room's corners (see Fig. 3 (e) and (f)).

Moreover, from Fig. 4, it is clear that the Lambertian order $m$ impacts the associated optimal LED locations as well. An elevated $m$, effectuates a left-shift of the vertex of the interLED-spread - $p_{10-90}$ curve towards smaller inter-LED-spreads i.e. it requires the inter-LED spread to shrink. This effect is noticeable for all 4 of the triangle, star, square and planned LED arrangement, and is more pronounced for $K=4$.

The conclusion made in Section IV-A5 for $K=3$ thus also extends to the considered Lambertian orders that vary from $m=1$. The LEDs are best arranged in a square, with the inter-LED-spread determined by $m$.

3) Interplay between $K$ and $N$

Figs. 3 to 5 all highlight that the optimal $p_{10-90}$ is reached for $K=3$ rather than for $K=4$, and this for multilateration with all considered combinations of a LED arrangement and a Lambertian order $m$. The more concentric error distribution of $K=4$ exhibiting larger errors is visualised in Fig. $3(\mathrm{~g})$ and (h). Fig. 6 shows the $p_{10-90}$ metrics associated with the LED layouts that are planned for an ascending number of LEDs $N$ and a varying $K$.

Fig. 6 clearly shows that $K=3$ is outperforming, in order, $K=4$ and $K=N$, for the ideal VLP room. From Fig. 6 the following can also be observed. (1) For $K \in[3,4]$, the $p_{10-90}$ gain associated with adding LEDs (i.e. increasing $N$ ) to the roll-out saturates. Bare in mind that this analysis does not include the performance degrading effect of additional 


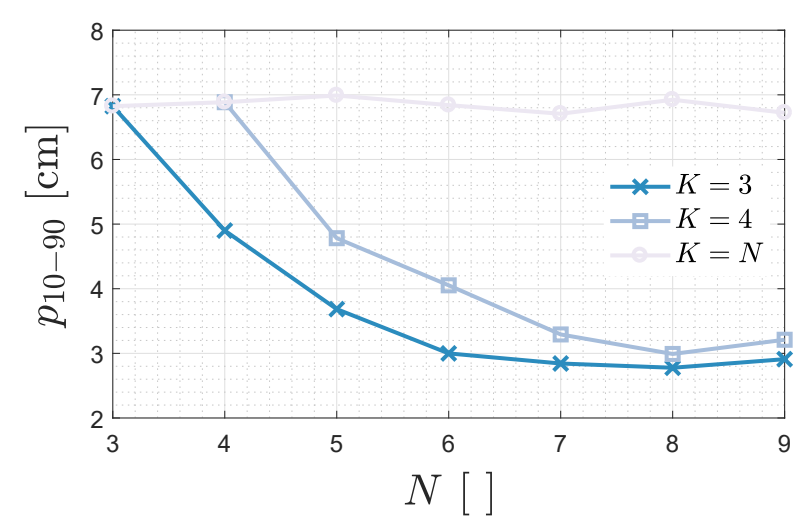

Fig. 6. $p_{10-90}$ as function of the number of LEDs $N$ for $K=3,4$ and $N$.

(demultiplexing) interference for a growing $N$ [12]. (2) The $p_{10-90}$ reduction when employing $K^{\prime}=K-1$ instead of $K$ dwindles with increasing $N$. (3) The $N-p_{10-90}$ curve for $K=N$ is approximately a horizontal line, meaning that adding more LEDs does not improve the positioning accuracy when all LEDs are used in the multilateration.

$K$ influences the GWO LED locations as well. For $K=N$, the GWO output LEDs flock to the corners of a triangle of comparable size. For $K=3$ and $N \in[4,5]$, the LED arrangement forms a quadrilateral and a quadrilateral with a near-centre LED, respectively. For $K=3$ and $5<N \leq 9$, the LEDs surround a single or two near-centre LED(s) depending on whether $N$ is even or odd. The surrounding LEDs are spread around to cover the positioning area. $K$ also significantly impacts the $p_{10-90}$ of square positioning lattices. In particular, in selecting $K$ LEDs, care must be taken to refrain from employing the $P_{R, i}$ of (near) collinear LEDs in the multilateration. For $K>3$, GWO LED arrangements should be favoured over squared ones.

\section{4) Influence of the Positioning Algorithm}

The (sub)optimal LED locations are contingent on the positioning algorithm employed. GWO is used to provide a LED arrangement for multilateration (shortened to Tril), for model-based fingerprinting (MBF) with $K=3$ and $K=4$ and with either a squared or normalised squared cost function [12] (denoted by respectively $M B F, M B F-4, M B F-N$ and $M B F$ $N$ - 4), for weighted least-squares trilateration (WLS) and for Cayley-Menger Determinant (CMD)-based localisation [49]. In the $M B F$-based algorithms, a $2.5 \mathrm{~cm}$ granularity is utilised. More details on the considered localisation algorithms can be found in previous work [12].

Fig. 7 graphically illustrates the relative and absolute $p_{10-90}$ when the algorithms on the left and at the bottom are combined for positioning and GWO planning respectively. The $p_{10-90}$ of each positioning algorithm is 1000 times averaged. As the best (relative) outcomes are found on the diagonal, Fig. 7 substantiates the $p_{10-90}$ improvement found when the employed positioning and planning algorithm are the same. For example, employing $M B F$ with a Tril LED arrangement comes at a significant relative cost of $9.2 \%$. The behaviour of $W L S$ and $C M D$ is equivalent to that of Tril, and is consequently omitted.

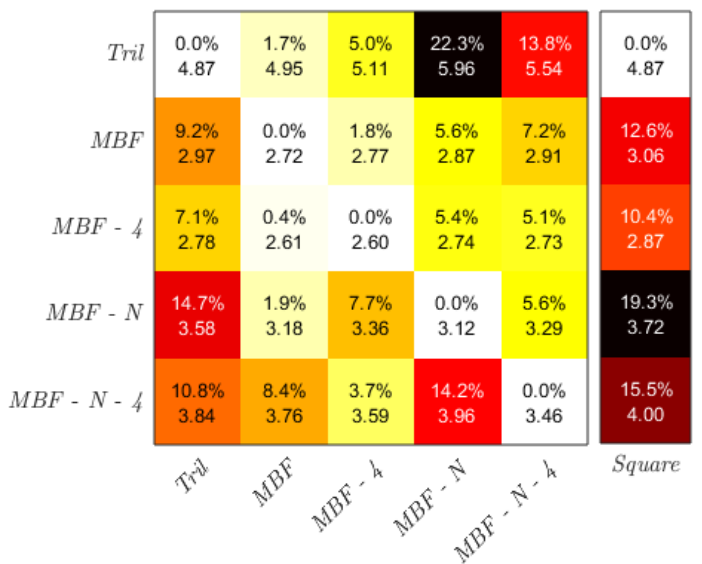

Fig. 7. The relative and absolute $p_{10-90}$ cost for when the algorithms on the left and at the bottom are respectively utilised for positioning and for $G W O$ planning. The $p_{10-90}$ of each algorithm in the presence of the LED square is provided at the right for reference.

From Fig. 7, it can also be derived that the square LED constellation, works well, relatively to the GWO arrangement, in conjunction with trilateration, but not with the MBF algorithms. In fact, the $M B F$ class achieves at least $10.4 \%-19.3 \%$ more accurate localisation with a dedicated constellation.

Fig. 7 thus proves that the (sub)optimal LED locations are arranged distinctively different for each positioning algorithm. Remarkably, for $K=4$ in conjunction with the $M B F$-class, the LEDs are arranged in a quadrilateral (shaped like $45^{\circ}$ Square), as opposed to a triangle-like shape for Tril.

\section{Influence of the Positioning Environment}

This Section IV-C investigates the $p_{10-90}$ gain of the GWO output (with $K=3$ ), with respect to the $2.5 \mathrm{~m}$ square, found when the parameters of the positioning environment vary. The impact of the room's dimensions, of the room shape, or of the introduction of diffuse or specular NLOS contributions is studied.

\section{1) Dimensions of the $2 D$ Positioning Ground Plane}

a) Enlarging the Square Ground Plane's side(s): Fig. 8 (a) depicts the exponential $p_{10-90}$ evolution for $m \in$ $[0.5,1,5]$ in response to a uniformly expanding square (in plane) positioning grid. Within bounds, smaller horizontal (i.e. $x y$ ) room dimensions pair better with LEDs with higher Lambertian orders. However, their steeper $P_{R, i}-d_{i}$ gradient has a larger exponential growth factor as a consequence. This growth manifests once a critical horizontal dimension is reached. Fig. 8 (a) also provides an inset that shows that the area enclosed by the LED locations rises to limit the exponential $p_{10-90}$ trend, as is intuitively expected.

b) Non-square Ground Planes: Symmetry-wise, square positioning grids still benefit square LED constellations. Unsurprisingly, when rooms with different shapes present themselves, e.g. a T-shape, GWO increasingly trumps the square LED arrangement. The GWO $p_{10-90}$ gain exceeds $1.2 \%$ for homolographic grids that are triangular, pentagonal or hexagonal in shape. Were the grid rectangular with a 1.5 dimension ratio (also with $25 \mathrm{~m}^{2}$ area), or were the lower triangle of the 


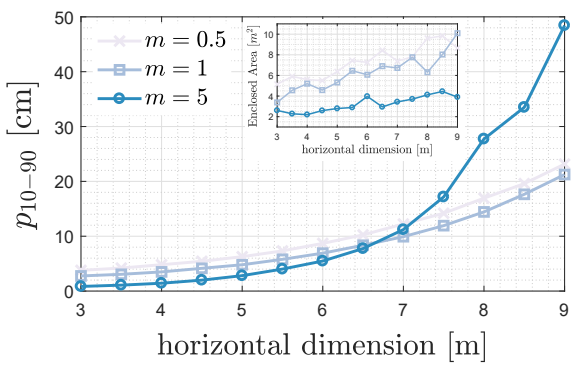

(a)

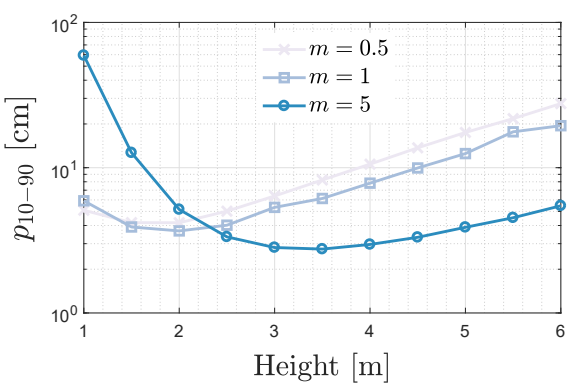

(b)

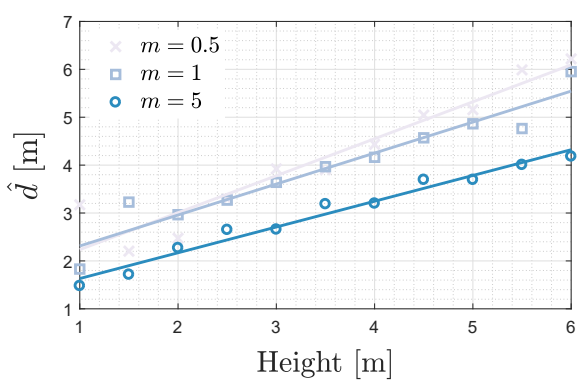

(c)

Fig. 8. Influence of the (a) horizontal and (b) vertical dimension of the positioning environment on the perceived $p_{10-90}$ for $m=0.5$, 1 and 5 . (c) demonstrates the LEDs moving apart, by means of $\hat{d}$ as a function of the LED suspension height (i.e. the vertical inter-LED-PD spread).

square grid inaccessible due to an obstacle, $G W O$ effectuates a $p_{10-90}$ improvement of over $8 \%$ and $48 \%$, respectively.

As long as the environment, potentially spanning multiple rooms, is characterised into the simulator tool, the $G W O$ planning tool is able to determine a (sub)optimal LED placement.

2) Influence of the LEDs' suspension height

The $p_{10-90}$ influence of the vertical dimension Height is illustrated in Fig. 8 (b) for $m=0.5,1$ and 5. It demonstrates that an optimal $m$ is coupled to each LED (suspension) height. The shorter vertical distances (between LEDs and PD) favour more omnidirectional radiation patterns i.e. with a lower Lambertian order. The heights of minimal $p_{10-90}$ equal $2 \mathrm{~m}$, $2 \mathrm{~m}$ and $3.5 \mathrm{~m}$ for $m=0.5, m=1$ and $m=5$, respectively. Industrial-like environments, characterised by heights larger than $3 \mathrm{~m}$, demand LEDs with $m>1$ to deliver $p_{10-90}<5 \mathrm{~cm}$ accurate localisation provided that the LED deployment is dense enough. With increasing Height, the LEDs move further apart as can be seen from the not normalised rms distance from centre $\hat{d}=\sqrt{\left(x_{S, i}-2.5 \mathrm{~m}\right)^{2}+\left(y_{S, i}-2.5 \mathrm{~m}\right)^{2}}$ growing approximately linearly (see Fig. 8 (c)). Actual environments impose a trade-off on $m$ in terms of coverage (favouring a smaller $m$ ) and localization gradient (higher $m$ ) to increase the accuracy.

\section{3) Planning in the presence of Non-Line-Of-Sight}

Importantly, the LED locations can also be honed to diminish the detrimental impact of Non-Line-Of-Sight (NLOS). The propagation models, detailed in Section III-A2, are wielded to study the effect of purely diffuse and specular reflections on the $p_{10-90}$, when localising in the presence of either a single wall (at $y=0$ ) or two adjacent walls (at $x=0$ and $y=0$ ). The uniform walls are divided in $20^{2}$ finite elements, and no higher order reflections are considered.

a) Purely Diffuse Reflections: Fig. 9 shows the $p_{10-90}$ for an accruing coefficient of reflection $\rho$ of the wall(s) for the square LED arrangement in red shades, for the best LOS $G W O$ solution (of Section IV-A1) in green shades, and in blue shades for LED locations where the GWO planning accounts for the specific NLOS case. $\rho=0$ coincides with the LOS case.

Expectedly, the $\rho-p_{10-90}$ trend is upward for all curves and the $p_{10-90}$ gap between the 1 wall and 2 walls configuration widens with a growing $\rho$. It can also be remarked that optimising the LED locations clearly reduces the detrimental

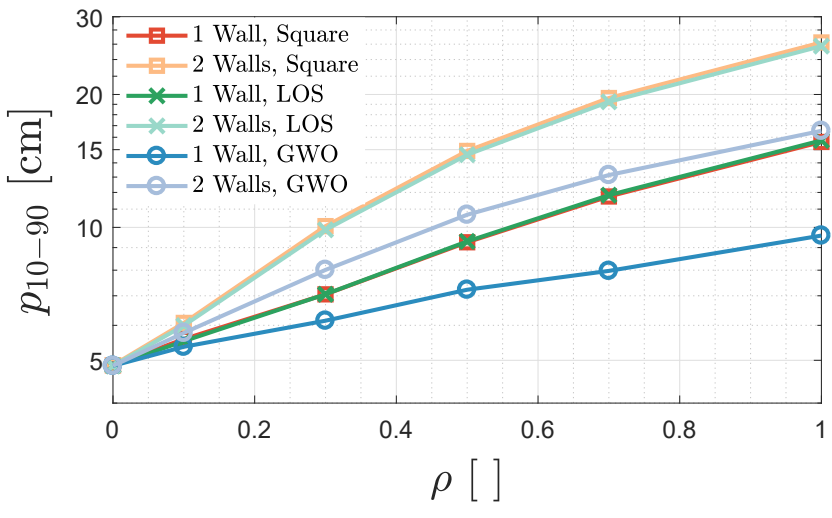

Fig. 9. $p_{10-90}$-influence of the diffuse reflections' coefficient of reflection.

impact of NLOS as a substantial $p_{10-90}$ benefit of $G W O$ over the square constellation is visible.

b) Specular Reflections: The influence of specular reflections on the other hand is characterised by Fig. 10, which displays the $p_{10-90}$ as a function of (a) the ratio that is diffusely reflected $r_{d}$ and (b) the specular Lambertian order $m_{s}$ for the single and double wall configuration with either $\rho=0.3$ and 0.7 .

Concordant with the findings for diffuse reflections, the main conclusion is again that sizeable $p_{10-90}$ reductions are found when $G W O$ optimising the LED locations, instead of using the square LED arrangement (in Fig. 10 denoted by $S Q$ ). Furthermore, from Fig. 10 (a), it can be derived that an increasing $r_{d}$ effectuates a decrease in $p_{10-90}$. In other words, the presence of specular reflections worsens the positioning accuracy even more than diffuse reflections do. This effect becomes more pronounced, when $\rho$ rises. The additional wall again adds to the $p_{10-90}$ error. The more minor influence of $m_{s}$ expectedly manifests mainly at smaller $r_{d}$. For $r_{d}=0$ or $r_{d}=0.2$, Fig. 10 (b) shows the rising $p_{10-90}$ evolution for $m_{s}=3$ to 24 as saturating (or already having obtained a maximum). For highly directive specular reflections, i.e. with larger $m_{s}$, the $p_{10-90}$ is expected to drop as the NLOS degradation will be more spatially confined.

Naturally, the associated GWO LED constellations are reflection-parameter and obstacle-type (1 wall vs 2 walls) dependent. For 1 wall, the LEDs tend to be arranged in a compressed and right-shifted version of Num. Sim. - 1 (see 


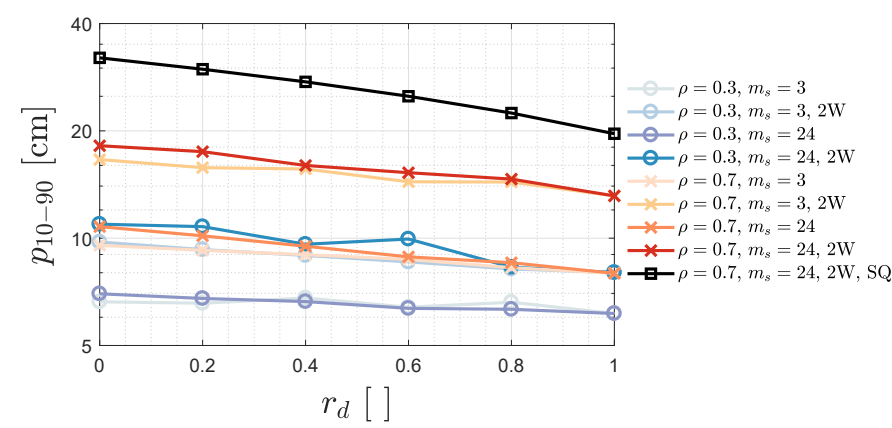

(a)

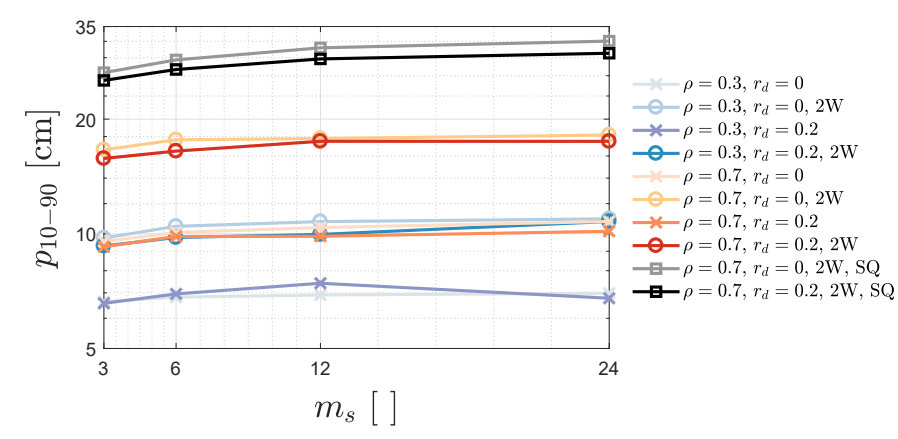

(b)

Fig. 10. Influence of the reflection parameters (a) $r_{d}$ and (b) $m_{s}$ on the $p_{10-90}$ for $\rho=0.3,0.7$ and for the 1 wall or 2 walls configuration (2W).

TABLE III

THE BEST, MEAN AND STANDARD DEVIATION ON THE, $p_{10-90}$ SCORE WHEN IDEAL AND/OR REAL-LIFE LEDS ARE PLANNED FOR AND/OR ACTUALLY USED, COMPUTED OVER A $5 \mathrm{~cm}$-SPACED POSITIONING GRID

\begin{tabular}{c|c|c|c|c|} 
Planned $\boldsymbol{m}$ & Actual $\boldsymbol{m}$ & Best $(\mathbf{c m})$ & Median $(\mathbf{c m})$ & SD $(\mathbf{c m})$ \\
\hline$m=1.14$ & $m=1.14$ & 4.51 & 4.55 & 0.03 \\
$m=1$ & $m=1.14$ & 4.51 & 4.60 & 0.07 \\
$m=1$ & $m=1$ & 4.78 & 4.87 & 0.08 \\
\hline
\end{tabular}

Fig. 17. In the two walls case, depending on the specular magnitude, the LEDs gather in a star-like constellation or a rotated trapezoid that is moved in direction of the room's top right.

\section{Towards Planning a Practical VLP roll-out}

So far, all LED and receiver characteristics were presumed ideal. This last subsection IV-D is devoted to examining the performance degrading effect of real-life non-idealities that practical deployments incur, with respect to the planned rollout.

\section{1) Impact of Radiation Pattern}

The radiation pattern of real-life, Lambertian-like, COB LEDs tends to diverge from the ideal Lambertian behaviour characterised by $m=1$. As a consequence, a bias is introduced when computing the ranges $d_{i}$ by inverting a notaccounting-for propagation model [21]. The bias on $d_{i}$ in turn worsens the trilateration $(K=3)$ accuracy.

The issue of 'actual' versus 'planned' (Lambertianapproximated) radiation pattern also presents itself during VLP network planning e.g. when changeover is required into an infrastructure planned for a different Lambertian order $m$. The question then rises whether the performance penalty is of that magnitude that the LED locations need to be changed.

In this simulation, $4 B X R E-35 E 2000-C-73$, each with an approximated $m=1.14$ [12], are replacing a $m=1$ LED constellation. The $p_{10-90}$ of the $m=1.14$ LEDs located according to the $m=1$ plan is compared to the $p_{10-90}$ found in a dedicated $m=1.14$ infrastructure. Both infrastructures of $m=1.14$ and $m=1$ are planned for using $10 \mathrm{GWO}$ algorithm runs. Table IIII lists the associated best and mean $p_{10-90}$ metric obtained for each of the three cases.
Table III allows deriving the following conclusions. A rollout planned for, and utilising $m=1.14$ outscores its $m=1$ counterpart (i.e. in line with Section IV-B3 by approximately $7 \%$, in terms of its mean $p_{10-90}$. When the latter $m=1$ infrastructure employs $m=1.14$ LEDs instead, its mean $p_{10-90}$ still ameliorates by $6 \%$. Hence, the penalty of not replanning when $m=1.14$ LEDs are used in an infrastructure planned for $m=1$ is limited to $1 \%$. As such, infrastructure changeover to a different Lambertian-like LED type (with not too much varying $m$ ) does not necessarily induce a significant $p_{10-90}$ increase and/or require repositioning the LED (sockets).

2) Impact of Placement Variations, Transmitter Tilt and (simultaneous) $P_{t, i}$ Calibration

Installation tolerances on the GWO-planned (or any other) LED locations are to be expected in practice [18]. Both mismatches on the LEDs' locations $\left(\Delta x_{S, i}, \Delta y_{S, i}, \Delta z_{S, i}\right)$ and non-zero LED tilt will be induced. This section quantifies the $p_{10-90}$ penalty incurred for various magnitudes of these mismatches.

Hereto, the mismatches around each LED are modelled as zero-mean Gaussians with standard deviation $\sigma_{\left(\Delta x_{S}, \Delta y_{S}\right)}$ (on the $x$ - and $y$-coordinate, in the LED plane) or $\sigma_{\Delta z_{S}}$ (on the $z$ coordinate). To generalise this analysis, the median positioning error $p_{50}$ is the metric considered for localisation on a $5 \mathrm{~cm}$ grid in the standard VLP roll-out (with the $2.5 \mathrm{~m}$ square).

Fig. 11 shows the $p_{50}$ as a function of (a) $\sigma_{\left(\Delta x_{S}, \Delta y_{S}\right)}$ and (b) $\sigma_{\Delta z_{S}}$ for Tril in green and $M B F(K=3)$ in black (refer to Section IV-B4 for algorithmic details). The $\sigma_{\left(\Delta x_{S}, \Delta y_{S}\right)}$ and $\sigma_{\Delta z_{S}}$ curves of both algorithms are exponential in nature, with the $p_{50}$ substantially increasing once the standard deviation mismatches (collected under the umbrella term $\sigma_{m}$ ) exceed $1 \mathrm{~cm}$. Moreover, a $\sigma_{m}=2 \mathrm{~cm}\left(\sigma_{m}=5 \mathrm{~cm}\right)$ already instills a $p_{50}$ rise of $13 \%(71 \%)$ and more. Upon installation, a $\sigma_{m} \leq 2 \mathrm{~cm}$ imposes that the 'maximum' (i.e. (99.7\%) 'peak') mismatch of $3 \sigma_{m}$ must not exceed $6 \mathrm{~cm}$. Fig. 11 also visualises that Tril is more sensitive to $\Delta z_{S}$, whereas for $M B F$ the $p_{50}$ onset is more pronounced for $x y$ mismatches.

In placing the LEDs, a normal distributed tilt with a $2^{\circ}$ standard deviation can easily be induced [18]. Localisation in the presence of uncompensated tilt is depicted in Fig. 11 and the corresponding curves have suffix $T$. Tilt introduces a $p_{50}$ surge during trilateration, while $M B F$ is more resistant. In 


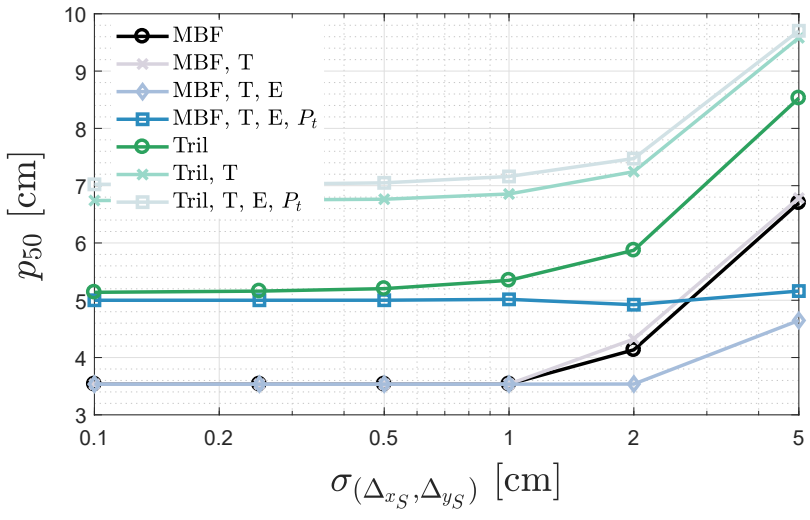

(a)

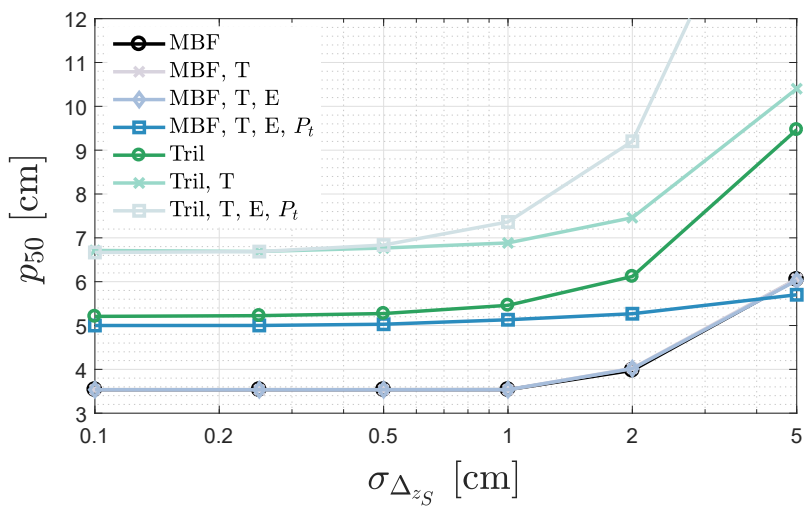

(b)

Fig. 11. Influence of installation-induced (a) $\sigma_{\left(\Delta x_{S}, \Delta y_{S}\right)}$-dictated $x y$ and (b) $\sigma_{\Delta z_{S}}$-dictated $z$ LED coordinate mismatches on the $p_{50}$ of Tril and $M B F$. The suffixes $T, E$ and $P_{t}$ denote the presence of transmitter tilt, tilt estimation and compensation, and $P_{t}$ calibration, respectively.

combination with $\sigma_{\left(\Delta x_{S}, \Delta y_{S}\right)}$, the by transmitter tilt induced $p_{50}$ increase is more pronounced.

The tilt contribution can for each LED be estimated, and in conjunction with $M B F$ (accounted for in the propagation model) be compensated, with the procedure from [50] (suffix $E)$. Interestingly, tilt compensation helps to alleviate the errors induced by $\sigma_{\left(\Delta x_{S}, \Delta y_{S}\right)}$, enlarging the $x y$ mismatch range for which $p_{50}<5 \mathrm{~cm}$ is obtained. In contrast, $\sigma_{\Delta z_{S}}$ hinders the tilt estimation/compensation.

If $P_{t, i}$ is not accurately known and it thus should be calibrated (suffix $P_{t}$ ) via $P_{R, i}$ (i.e. via the $P_{R, i}$ of the positioning grid closest to $\operatorname{LED}_{i}[20]$ ), a Tril $p_{50}$ penalty will ensue. With Tril, for $\sigma_{\left(\Delta x_{S}, \Delta y_{S}\right)}$ and $\sigma_{\Delta z_{S}}$, the additional $P_{t, i}$ calibration comes at a cost for smaller and larger $\sigma_{m}$, respectively. For $M B F$, effectuating a radiant power calibration (partly) reduces the combination impact of tilt and the LED coordinate mismatch, at larger $\sigma_{m}$.

\section{3) Impact of FOV}

The receiver's (single-sided) field-of-view (FOV) $\left|\Psi_{C}\right|$ in practice not attaining the ideal $90^{\circ}$, also impacts the attainable $p_{10-90}$ and spatial distribution of the optimal LED locations. For 4 LEDs, a decreasing $\left|\Psi_{C}\right|$ leads to an exponential $p_{10-90}$ increase. Spatially, for $K=3$, with a decreasing $\left|\Psi_{C}\right|$, the area between the planned LED locations shrinks as well i.e. up to the critical $\left|\Psi_{C}\right|$.

\section{EXPERIMENTAL RESULTS}

By means of the VLP measurement setup (detailed in Section III-C, this Section V compares the simulation-based positioning errors of the previous Section $[\mathrm{IV}$ with experimental data. Localisation data were collected with both the GWO planned and the traditional $2.5 \mathrm{~m}$ 'square' (denoted by $S Q$ ) LED constellation. As the LEDs were hung manually (and in a one-shot manner), the actual (and measured) LED locations differ (slightly) from the intended ones and small LED tilts are induced.

The measured positioning error cumulative distribution functions (CDFs) of $S Q$ and $G W O$, obtained with $K=3, m=$ 1 and averaged across 2 positioning runs, are visualised in Fig. 12 in the darkest green and darkest blue, respectively.

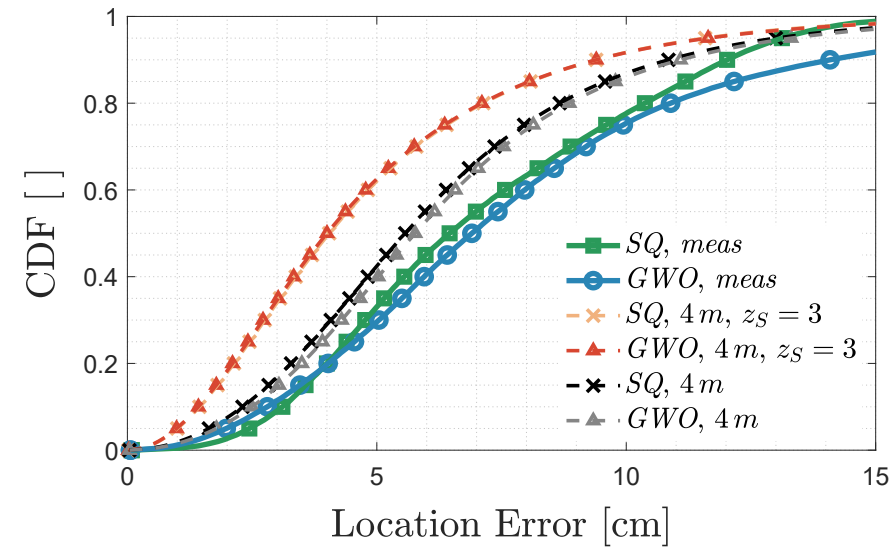

Fig. 12. Averaged Cumulative Distribution Functions (CDFs) of the positioning/location error found in simulation and in measurement, when localising with the $G W O$ and square $S Q$ LED configuration.

TABLE IV

$p_{10-90}, p_{50}, p_{75}$ AND $p_{90}$ VALUES ASSOCIATED WITH FIG. 12

\begin{tabular}{c|c|c|c|c|} 
Description & $\boldsymbol{p}_{\mathbf{1 0}-\mathbf{9 0}}[\mathrm{cm}]$ & $\boldsymbol{p}_{\mathbf{5 0}}[\mathrm{cm}]$ & $\boldsymbol{p}_{\mathbf{7 5}}[\mathrm{cm}]$ & $\boldsymbol{p}_{\mathbf{9 0}}[\mathrm{cm}]$ \\
\hline SQ, meas & 5.51 & 6.46 & 9.60 & 12.02 \\
$\boldsymbol{G W O}, \boldsymbol{m e a s}$ & 5.81 & 6.91 & 9.94 & 14.08 \\
\hline$S Q, 4 \mathrm{~m}, z_{S}=3 \mathrm{~m}$ & 3.53 & 4.05 & 6.40 & 9.40 \\
$G W O, 4 \mathrm{~m}, z_{S}=3 \mathrm{~m}$ & 3.50 & 4.00 & 6.36 & 9.40 \\
\hline$S Q, 4 \mathrm{~m}$ & 4.66 & 5.57 & 7.95 & 10.85 \\
$G W O, 4 \mathrm{~m}$ & 4.82 & 5.77 & 8.14 & 11.09 \\
\hline
\end{tabular}

The associated $p_{10-90}$ values amount to $5.51 \mathrm{~cm}$ and $5.81 \mathrm{~cm}$. Prior to the localisation, $P_{t, i}$ is calibrated at the grid point closest to the LED's location (see Section IV-D2). With the set LED driving current magnitude and the variations thereon, $P_{t, i} \neq P_{t}\left(P_{t}=10 \mathrm{~W}\right), i=1 . .4[12]$.

Simulations of the measurement setups (i.e. with the actual LED positions and a $2.5 \mathrm{~cm}$ grid) lead to the 1000 times averaged CDFs displayed in black (denoted by ' $S Q, 4 \mathrm{~m}^{\text {') }}$ and grey (denoted by ' $G W O, 4 m^{\prime}$ ). With the simulation $p_{10-90}$ equalling $4.66 \mathrm{~cm}$ and $4.82 \mathrm{~cm}$, both measurements and simulations agree on the conclusion that the actual $S Q$ rollout outperforms GWO's. This conclusion seems contradictory 
when reverting to Section IV-A2, were it not for the actual $z_{S, i}$ slightly diverging from the nominal $3 \mathrm{~m}$ value i.e. a $\sigma_{\Delta z_{S}}>0$ is present. By virtue of a comparison with 2 more simulation CDFs (in the red shades), where $z_{S, i}$ was set to $3 \mathrm{~m}$, it turns out that the square layout is more robust to $\sigma_{\Delta z_{S}}$ than the planned layout. With $\sigma_{\Delta z_{S}}=0, G W O$ does trump $S Q$ albeit marginally. Though the variations on the LEDs' $z_{S, i}$ are in the order of $1-2 \mathrm{~cm}$, these induce $p_{10-90}$ increases of more than $1 \mathrm{~cm}$ (in line with Section IV-D2). The robustness of a LED configuration is herewith proven to be an important area for further study. The future $G W O$ tool should hence also evaluate LED configurations with $\sigma_{m}$, to fully reap the planning benefits that are largest in the presence of NLOS.

The approximately $20 \%$ difference between ' $S Q$, $4 \mathrm{~m}^{\prime} /{ }^{\prime} G W O, 4 \mathrm{~m}$ ' and the measured $S Q / G W O p_{10-90}$ can be attributed to $P_{t, i}$ calibration, tilt, LED interference [12] and measurement impreciseness. These factors make it difficult to unambiguously discern the experimental $p_{10-90}$ gain of 2 LED layouts that are close in their expected $p_{10-90}$. All the CDF's $p_{10-90}, p_{50}, p_{75}$ and $p_{90}$ values are collected in Table IV

\section{CONCLUSION}

This work selected the Grey Wolf Optimizer (GWO) evolutionary algorithm for planning the (sub)optimal placement of VLP-enabled LEDs in the presence of various positioning parameters and environments. With simulations, it showed the (potential) $p_{10-90}$ gain obtained when the LED transmitters are carefully placed. The optimal LED arrangement depends on the LEDs' characteristics, the environment and the positioning parameters. Experimental verification of the simulations was performed in our $4 \mathrm{~m}$ by $4 \mathrm{~m}$ by $3 \mathrm{~m}$ lab.

\section{A. Guidelines}

The following guidelines when panning a VLP roll-out can be drawn up:

- When applying 2D multilateration in a strict Line-OfSight (LOS) environment, it is beneficiary to employ $K=3$ and to place the 4 LEDs in a square with an interLED-spread optimised for the LEDs' Lambertian order $m$. Higher $m$ LEDs favour smaller LED separations.

- Room width and height respectively negatively and positively correlate with $m$ (within bounds).

- LED locations optimised for multilateration are not necessarily optimal for other positioning algorithms. It is not the case for the more accurate (higher latency) class of model-based fingerprinting algorithms.

- Equipping a room with more VLP-enabled LEDs not necessarily improves the positioning performance. For multilateration, $K=3$ optimises the $p_{10-90}$, even when more than 4 LEDs are used.

- The GWO network planning tool shows its value when the positioning environment is non-square or when (significant) Non-Line-Of-Sight (NLOS) is present as the optimal LED layout is no longer square then.

- The VLP LED anchors need to be mindfully installed, as typically occurring variations or imperfections degrade the roll-out's positioning performance significantly.

\section{B. Future Work}

This work provides a stepping stone on the way to the development of a industrial-ready VLP network planner. Other required stepping stones comprise planning results in case of localisation in 3 dimensions, with arbitrary LEDs, in practical environments and with different positioning paradigms (e.g. with angle-of-arrival instead of received signal strengthbased VLP). Finally, a joint optimisation of communication/positioning and illumination, and its integration in the existing WHIPP network planner [51] is part of the future efforts.

\section{REFERENCES}

[1] T. Cogalan and H. Haas, "Why would 5G need optical wireless communications?," IEEE 28th Annual International Symposium on Personal, Indoor, and Mobile Radio Communications (PIMRC), Montreal, Canada, 2017, pp. 1-6, doi: 10.1109/PIMRC.2017.8292749.

[2] M. Kavehrad, "Sustainable energy-efficient wireless applications using light," in IEEE Communications Magazine, vol. 48, no. 12, pp. 66-73, 2010, doi: 10.1109/MCOM.2010.5673074.

[3] M. Z. Chowdhury, M. Shahjalal, M. K. Hasan and Y. M. Jang, "The Role of Optical Wireless Communication Technologies in $5 \mathrm{G} / 6 \mathrm{G}$ and IoT Solutions: Prospects, Directions, and Challenges," in Applied Sciences, vol. 9, no. 4367, pp. 1-20, 2019, doi: 10.3390/app9204367.

[4] M. B. Rahaim, A. M. Vegni and T. D. C. Little, "A hybrid Radio Frequency and broadcast Visible Light Communication system," IEEE GLOBECOM Workshops (GC Wkshps), Houston, TX, 2011, pp. 792 796, doi: 10.1109/GLOCOMW.2011.6162563.

[5] 5G PPP, "Internet of Radio Light (IORL)," https://iorl.5g-ppp.eu/ (accessed January 1, 2021).

[6] J. Cosmas, Y. Zhang and X. Zhang, "Internet of Radio-Light: 5G Broadband in Buildings," 23th European Wireless 2017 Conference, Dresden, Germany, 2017, pp. 74-79.

[7] J. Cosmas et al., "A Scalable and License Free 5G Internet of Radio Light Architecture for Services in Homes \& Businesses," IEEE International Symposium on Broadband Multimedia Systems and Broadcasting (BMSB), Valencia, Spain, 2018, pp. 1-6, doi: 10.1109/BMSB.2018.8436938.

[8] J. Cosmas et al., "5G Internet of radio light services for Musée de la Carte à Jouer," Global LIFI Congress (GLC), Paris, France, 2018, pp. 1-6, doi: 10.23919/GLC.2018.8319095.

[9] J. Cosmas et al., "5G Internet of radio light services for supermarkets," 14th China International Forum on Solid State Lighting: International Forum on Wide Bandgap Semiconductors China (SSLChina: IFWS), Beijing, China, 2017, pp. 69-73, doi: 10.1109/IFWS.2017.8245977.

[10] L. Shi et al., "5G Internet of Radio Light Positioning System for Indoor Broadcasting Service," in IEEE Transactions On Broadcasting, vol. 66, no. 2, pp. 534-544, 2020, doi: 10.1109/TBC.2020.2981755.

[11] B. Meunier and J. Cosmas, "5G Internet of Radio Light Virtual Reality System," IEEE International Symposium on Broadband Multimedia Systems and Broadcasting (BMSB), Valencia, Spain, 2018, pp. 1-5, doi: 10.1109/BMSB.2018.8436602.

[12] S. Bastiaens, K. Deprez, L. Martens, W. Joseph and D. Plets, "A Comprehensive Study on Light Signals of Opportunity for Subdecimetre Unmodulated Visible Light Positioning," in Sensors, vol. 20, no. 5596, pp. 1-27, 2020, doi: 10.3390/s20195596.

[13] Z. Li, A. Yang, H. Lv, L. Feng, W. Song, "Fusion of Visible Light Indoor Positioning and Inertial Navigation Based on Particle Filter," in IEEE Photonics Journal, vol. 9, pp. 1-13, 2017, doi: 10.1109/JPHOT.2017.2733556.

[14] F. Alam, N. Faulkner, M. Legg and S. Demidenko, "Indoor Visible Light Positioning Using Spring-Relaxation Technique in Real-World Setting," in IEEE Access, vol. 7, pp. 91347-91359, 2019, doi: 10.1109/ACCESS.2019.2927922.

[15] Interact, "Indoor navigation and location analytics," https: //www.interact-lighting.com/global/what-is-possible/interact-retail/ indoor-navigation (accessed January 1, 2021).

[16] S. Cincotta, C. He, A. Neild, J. Armstrong, "Indoor Visible Light Positioning: Overcoming the Practical Limitations of the Quadrant Angular Diversity Aperture Receiver (QADA) by Using the Two-Stage QADA-Plus Receiver,' in Sensors, vol. 19, no. 956, pp. 1-23, 2019, doi: 10.3390/s19040956. 
[17] N. Stevens and H. Steendam, "Influence of Transmitter and Receiver Orientation on the Channel Gain for RSS Ranging-Based VLP," 11th International Symposium on Communication Systems, Networks \& Digital Signal Processing (CSNDSP), Budapest, Hungary, 2018, pp. 1-5, doi: 10.1109/CSNDSP.2018.8471749.

[18] D. Plets, S. Bastiaens, L. Martens and W. Joseph, "An Analysis of the Impact of LED Tilt on Visible Light Positioning Accuracy," in Electronics, vol. 8, no. 389, pp. 1-15, 2019, doi: 10.3390/electronics8040389.

[19] S. Bastiaens, D. Plets, L. Martens and W. Joseph, "Impact of Nonideal LED Modulation on RSS-based VLP Performance," IEEE 29th Annual International Symposium on Personal, Indoor and Mobile Radio Communications (PIMRC), Bologna, Italy, 2018, pp. 1-5, doi:10.1109/PIMRC.2018.8581009.

[20] D. Plets, S. Bastiaens, L. Martens, W. Joseph, and N. Stevens, "On the impact of LED power uncertainty on the accuracy of 2D and 3D visible light positioning," in OPTIK, vol. 195, pp. 1-12, 2019, doi: 10.1016/j.ijleo.2019.163027.

[21] N. Stevens, "Bias Introduced by True Radiation Patterns in RSS-based Visible Light Positioning," IEEE SENSORS, Montreal, Canada, 2019, pp. 1-4, doi: 10.1109/SENSORS43011.2019.8956584

[22] C. Gong, "Visible Light Communication and Positioning: Present and Future," in Electronics, vol. 8, no. 788, pp. 1-3, 2019, doi: 10.3390/electronics 8070788 .

[23] G. Shi, Y. Li, W. Cheng, L. Dong, J. Yang and W. Zhang, "Accuracy analysis of indoor visible light communication localization system based on received signal strength in non-line-of-sight environments by using least squares method," in Optical Engineering, vol. 5, no. 5, 2019, pp. 1-11, doi: 10.1117/1.OE.58.5.056102.

[24] H. Steendam, "A 3-D Positioning Algorithm for AOA-Based VLP With an Aperture-Based Receiver," in IEEE Journal on Selected Areas in Communications, vol. 36, no. 1, pp. 23-33, 2018, doi: 10.1109/JSAC.2017.2774478.

[25] S. Jung, S. Hann and C. Park, "TDOA-based optical wireless indoor localization using LED ceiling lamps," in IEEE Transactions on Consumer Electronics, vol. 57, no. 4, pp. 1592-1597, 2011, doi: 10.1109/TCE.2011.6131130.

[26] D. Plets, Y. Almadani, S. Bastiaens, M. Ijaz, L. Martens, and W. Joseph, "Efficient 3D trilateration algorithm for visible light positioning," in Journal Of Optics, vol. 21, no. 5, pp. 1-4, 2019.

[27] S. K. Goudos, Evolutionary Algorithms for Wireless Communications A Review of the State-of-the art. InTech, 2014, ch. 1, pp. 1-24, doi: $10.5772 / 59147$

[28] J. Kennedy and R. Eberhart, "Particle Swarm Optimization," in IEEE International Conference on Neural Networks, Piscataway, NJ, 1995, pp. 1942-1948, doi: 10.1109/ICNN.1995.488968.

[29] S. Mirjalili, S. M. Mirjalili, and A. Lewis, "Grey wolf optimizer," in Advances in Engineering Software, vol. 69, pp. 46-61, 2014, doi: 10.1016/j.advengsoft.2013.12.007.

[30] R. V. Rao, "Jaya : A simple and new optimization algorithm for solving constrained and unconstrained optimization problems," in International Journal of Industrial Engineering Computations, vol. 7, no. 1, pp. 1934, 2016, doi: 10.5267/j.ijiec.2015.8.004

[31] S. Mirjalili, A. H. Gandomi, S. Z. Mirjalili, S. Saremi, H. Faris, and S. M. Mirjalili, "Salp Swarm Algorithm: A bio-inspired optimizer for engineering design problems," in Advances in Engineering Software, 2017, doi: 10.1016/j.advengsoft.2017.07.002

[32] S.-A. Chu, P.-W. Tsai, and J.-S. Pan, "Cat swarm optimization," Lecture Notes in Computer Science (including subseries Lecture Notes in Artificial Intelligence and Lecture Notes in Bioinformatics), vol. 4099 LNAI, pp. 854-858, 2006, doi: 10.1007/978-3-540-36668-3_94.

[33] J. Ding, I. Chih-Lin, H. Zhang, X. Chen, B. Yu and H. Lai, "Cells Planning of VLC Networks using Non-Circular Symmetric Optical Beam," IEEE International Conference on Communications (ICC), Shanghai, China, 2019, pp. 1-6, doi: 10.1109/ICC.2019.8761098.

[34] S. Pergoloni, M. Biagi, S. Colonnese, R. Cusani and G. Scarano, "Optimized LEDs Footprinting for Indoor Visible Light Communication Networks," in IEEE Photonics Technology Letters, vol. 28, no. 4, pp. 532-535, 2016, doi: 10.1109/LPT.2015.2500360.
[35] I. Stefan and H. Haas, "Analysis of Optimal Placement of LED Arrays for Visible Light Communication," IEEE 77th Vehicular Technology Conference (VTC Spring), Dresden, Germany, pp. 1-5, 2013.

[36] A. Singh, A. Srivastava, V. A. Bohara and G. S. V.R.K. Rao, "Performance of Indoor Visible Light Communication System Under Random Placement of LEDs," 21st International Conference on Transparent Optical Networks (ICTON), Angers, France, 2019, pp. 1-5, doi: 10.1109/ICTON.2019.8840427.

[37] C. You, S. Zhang and R. Zhang, "An optimal LED light source arrangement to reduce SNR fluctuation for MISO VLC system using fireworks algorithm," International Journal of Grid and Distributed Computing, vol. 9, no. 12, pp. 295-306, 2013, doi: 10.14257/ijgdc.2016.9.12.26.

[38] M. Ajmani, S. Sinanović and T. Boutaleb, "Optimal beam radius for LED-based indoor positioning algorithm," International Conference for Students on Applied Engineering (ICSAE), Newcastle upon Tyne, UK, 2016, pp. 357-361, doi: 10.1109/ICSAE.2016.7810217.

[39] D. Wu et al., "Effect of Optimal Lambertian Order for Cellular Indoor Optical Wireless Communication and Positioning Systems", in Optical Engineering, vol. 55, no. 6, pp. 1-8, 2016, doi: 10.1117/1.OE.55.6.066114.

[40] D. Plets et al., "Three-dimensional Visible Light Positioning: an Experimental Assessment of the Importance of the LEDs' Location," International Conference on Indoor Positioning and Indoor Navigation (IPIN) 2019, Pisa, Italy, 2019, pp. 1-6, doi: 10.1109/IPIN.2019.8911763.

[41] Y. Almadani, M. Ijaz, S. Rajbhandari, U. Raza and B. Adebisi, "DeadZones Limitation in Visible Light Positioning Systems for Unmanned Aerial Vehicles," Eleventh International Conference on Ubiquitous and Future Networks (ICUFN), Zagreb, Croatia, 2019, pp. 419-421, doi: 10.1109/ICUFN.2019.8806081.

[42] S. De Lausnay, L. De Strycker, J. P. Goemaere, N. Stevens and B. Nauwelaers, "A Visible Light Positioning system using Frequency Division Multiple Access with square waves," International Conference on Signal Processing and Communication Systems, Cairns, QLD, 2015, pp. 1-7, doi: 10.1109/ICSPCS.2015.7391787.

[43] W. Gu, M. Aminikashani, P. Deng and M. Kavehrad, "Impact of Multipath Reflections on the Performance of Indoor Visible Light Positioning Systems," in Journal of Lightwave Technology, vol. 34, pp. 2578-2587, 2016, doi: 10.1109/JLT.2016.2541659..

[44] J. M. Kahn and J. R. Barry, "Wireless infrared communications," in Proceedings of the IEEE, vol. 85, no. 2, pp. 265-298, 1997, doi: $10.1109 / 5.554222$

[45] C. R. Lomba, R. T. Valadas and A. M. de Oliveira Duarte, "Experimental characterisation and modelling of the reflection of infrared signals on indoor surfaces," in IEE Proceedings - Optoelectronics, vol. 145, no. 3 , 1998, pp. 191-197, doi: 10.1049/ip-opt:19982020.

[46] J.O. Roa, A. R. Jiménez, F. Seco, J. Ealo, C. Prieto, and J. Guevara, "Optimal placement of sensors in trilateration: Regular lattices vs meta-heuristic solutions," International Conference on Computer Aided Systems Theory, Las Palmas de Gran Canaria, Spain, February 12-16, 2007, pp. 1-3, doi: 10.1007/978-3-540-75867-9_98.

[47] T. Komine and M. Nakagawa, "Fundamental analysis for visiblelight communication system using LED lights," in IEEE Transactions on Consumer Electronics, vol. 50, no. 1, pp. 100-107, 2004, doi: 10.1109/TCE.2004.1277847.

[48] Y. Zhuang et al., "Visible Light Positioning and Navigation Using Noise Measurement and Mitigation," in IEEE Transactions on Vehicular Technology, vol. 68, no. 11, pp. 11094-11106, 2019, doi: 10.1109/TVT.2019.2943517.

[49] F. Thomas and L. Ros, "Revisiting trilateration for robot localization," in IEEE Transactions on Robotics, vol. 21, no. 1, pp. 93-101, 2005, doi: $10.1109 /$ TRO.2004.833793.

[50] S. Bastiaens, W. Raes, N. Stevens, L. Martens, W. Joseph and D. Plets, "Impact of a Photodiode's Angular Characteristics on RSS-Based VLP Accuracy," in IEEE Access, vol. 8, pp. 83116-83130, 2020, doi: 10.1109/ACCESS.2020.2991298.

[51] D. Plets, W. Joseph, K. Vanhecke, E. Tanghe and L. Martens, "Coverage prediction and optimization algorithms for indoor environments," in EURASIP Journal on Wireless Communications and Networking, vol. 123, pp 1-23, 2012, doi: 10.1186/1687-1499-2012-123. 